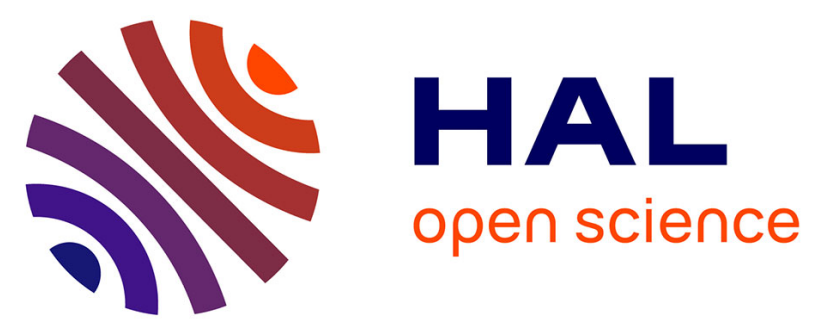

\title{
On-line determination of nanometric and sub-micrometric particle physicochemical characteristics using spectral imaging-aided Laser-Induced Breakdown Spectroscopy coupled with a Scanning Mobility Particle Sizer
}

Tanguy Amodeo, Christophe Dutouquet, Olivier Le Bihan, Michel Attoui, Emeric Frejafon

\section{- To cite this version:}

Tanguy Amodeo, Christophe Dutouquet, Olivier Le Bihan, Michel Attoui, Emeric Frejafon. Online determination of nanometric and sub-micrometric particle physicochemical characteristics using spectral imaging-aided Laser-Induced Breakdown Spectroscopy coupled with a Scanning Mobility Particle Sizer. Spectrochimica Acta Part B: Atomic Spectroscopy, 2009, 64 (10), pp.1141-1152. 10.1016/j.sab.2009.07.031 . ineris-00961943

\section{HAL Id: ineris-00961943}

https://hal-ineris.archives-ouvertes.fr/ineris-00961943

Submitted on 20 Mar 2014

HAL is a multi-disciplinary open access archive for the deposit and dissemination of scientific research documents, whether they are published or not. The documents may come from teaching and research institutions in France or abroad, or from public or private research centers.
L'archive ouverte pluridisciplinaire HAL, est destinée au dépôt et à la diffusion de documents scientifiques de niveau recherche, publiés ou non, émanant des établissements d'enseignement et de recherche français ou étrangers, des laboratoires publics ou privés. 
On-line determination of nanometric and sub-micrometric particle physicochemical characteristics using Spectral Imaging-aided Laser-Induced Breakdown Spectroscopy coupled with a Scanning Mobility Particle Sizer.

Tanguy Amodeo ${ }^{\mathrm{a}}$, Christophe Dutouquet ${ }^{\mathrm{a}}$, Olivier Le Bihan ${ }^{\mathrm{a}}$, Michel Attoui $^{\mathrm{b}}$ and Emeric Frejafon $^{\mathrm{a}}$

a Institut National de l'Environnement Industriel et des Risques (INERIS), Parc Technologique Alata, BP2, 60550 Verneuil-En-Halatte, France

tanguy.amodeo@ineris.fr, christophe.dutouquet@ineris.fr, olivier.le-bihan@ineris.fr, emeric.frejafon@ineris.fr

${ }^{\text {b }}$ Université Paris XII, département de physique, Faculté des Sciences et Technologie, France attoui@univ-paris12.fr

\section{$\underline{\text { Corresponding author: }}$}

Christophe Dutouquet

Phone: +33 (0) 344618146

Fax: + $33(0) 344556302$

Email address: christophe.dutouquet@ineris.fr 


\begin{abstract}
Laser-Induced Breakdown Spectroscopy (LIBS) has been employed to detect sodium chloride and metallic particles with sizes ranging from $40 \mathrm{~nm}$ up to $1 \mu \mathrm{m}$ produced by two different particle generators. The LIBS technique combined with a Scanning Mobility Particle Sizer (SMPS) was evaluated as a potential candidate for workplace surveillance in industries producing nanoparticle-based materials. Though research is still currently under way to secure nanoparticle production processes, the risk of accidental release is not to be neglected. Consequently, there is an urgent need for the manufacturers to have at their command a tool enabling leak detection in-situ and in real time so as to protect workers from potential exposure.

In this context, experiments dedicated to laser-induced plasma particle interaction were performed. To begin with, spectral images of the laser-induced plasma vaporizing particles were recorded to visualize the spatiotemporal evolution of the atomized matter and to infer the best recording parameters for LIBS analytical purposes, taking into account our experimental set-up specificity. Then, on this basis, time-resolved spectroscopic measurements were performed to make a first assumption of the LIBS potentialities. Particle size dependency on the LIBS signal was examined. Repeatability and limits of detection were assessed and discussed. All the experiments carried out with low particle concentrations point out the high time delays corresponding to the LIBS signal emergence. Plasma temperature temporal evolution was found to be a key parameter to explain this peculiarity inherent to laser / plasma / particle interaction.
\end{abstract}

\title{
Keywords
}

LIBS, Plasma imagery, on-line monitoring, workplace surveillance, nanoparticles, 


\section{Introduction}

Today, nanotechnology lets foresee many opportunities and benefits for new materials with significantly improved properties as well as revolutionary applications in large industrial fields. Indeed, materials designed at a nanometric scale are expected to show new properties of great interest to a wide range of industrial applications. In this context, the development of nanoparticle-based materials has advanced rapidly and along with it the need for safe nanoparticle production systems. Nanoscale materials find applications in numerous fields such as electronics, materials, energy, pharmaceutical and the list is far from being exhaustive.

Nanotechnology is often said to be the industry of the $21^{\text {st }}$ century and its future development is expected to grow in years to come. Analysts belonging to the world of economics estimate that the worldwide marketplace related to the nanotechnology industry will be worth around 1000 billion euro by 2011 [1]. Production and use of nanoparticles are already key technologies in the EU's strategy of strengthening the European Research Area. The apparent limitless possibilities offered by nanotechnologies in terms of applications and economic gain are to lead to a dramatic increase of engineered nanoparticle production. Such a fast development raises the issue of the security of the personnel working in the production plants. Though research on methods intended to secure the production processes have been carried out and are still currently under way, the possibility of accidental nanoparticle release is not to be neglected. As a consequence, there is a matter of urgency to assess the risks associated to nanoparticle exposure. With the emergence of nanoparticlebased materials, concerns regarding health and safety have been brought to the fore.

Little is known yet about potential health effects nanoparticles might induce. Due to their small sizes, nanoparticles are more likely to get past biological barriers and penetrate much deeper into organs (e.g. the lungs) than their micrometric counterparts. Inhalation, ingestion and skin absorption are possible ways of penetration through the human body. The potential dangers of the engineered nanoparticles stem from their unique physical and chemical properties conferring them specific properties in the way they interact with biological systems. Nanoparticle characteristics such as chemical composition, surface area, solubility may influence toxicity [2].

Besides potential health effects, risks pertained to fire and explosion come also under scrutiny. With their increased surface, less energy is required to trigger an explosion than that needed for larger particles. Moreover, nanoparticle oxidation is likely to take place with faster reaction kinetics than with coarser particles thereby leading to a higher explosion probability if released accidentally in the ambient air. For both potential 
health and safety matters, research on these topics is still currently under way to pin down possible noxious effects among all those potentially existing.

All these potential risks point out the insistent need to monitor and secure the workplace to minimize hazards in production plants where nanoparticles are being manufactured, stored, transported, handled, applied and used in different processes, products, conditions and environments. Thus, the NANOSAFE2 project funded by the EU within the $6^{\text {th }}$ framework program (FP6) was set up to elaborate solutions to make production processes and use of nanomaterials safer [3]. The project mainly aims at developing safe industrial production systems as well as detection, monitoring and characterization techniques. It also includes health and hazard assessment studies along with research on environmental and societal aspects connected to nanomaterial production and use.

Several reports and articles [4] have emphasized the lack of appropriate metrology tools for workplace surveillance. At present time, several expensive and sophisticated off-line systems exist such as microscopic techniques (STEM, STM or PEEM) giving structural information that can be combined with electron and X-ray diffraction for information on compositions (RHEED, LEED, XRD). There are also in-situ spectroscopy (ATR, Raman, GD-OES) or X-rays photoelectron spectroscopy (XPS), Auger electron spectroscopy (AES), photoelectron emission spectroscopy and photon correlation spectroscopy (PCS) but most of them suffer of high detection limit and might depend on nanoparticle shape or size. As a consequence, there is currently no device that directly measures worker exposure to, and the toxicity of, engineered nanoparticles, with monitoring being limited to expensive laboratory analysis of ambient air.

Accordingly, an on-line tool dedicated to nanoparticle monitoring had to be designed to protect the work place atmosphere [1]. Thus, under the umbrella of the NANOSAFE2 program, the implementation of an on-line monitoring technique allowing determination of both chemical and physical nanoparticle characteristics in air media was envisaged. The retained solution lies in the combining of the Laser Induced Breakdown Spectroscopy Technique (LIBS) with a commercially available Differential Mobility Analyses (DMA) connected to a Condensation Particle Counter (CNC), the latter two devices constituting the Scanning Mobility Particle Sizer (SMPS). Nanoparticle chemical and physical characteristics are then obtained from the LIBS system and the SMPS respectively.

LIBS has proved to be a versatile, reliable analytical method for multi-elemental analysis of solid [5], liquid [6], gas [7,8] and aerosols [9,10] both for qualitative and quantitative measurements. It has the advantage of being non-intrusive and no sampling is required. Moreover, measurements can be performed at remote 
distances, in real time and in-situ without sample preparation. Several studies have demonstrated the LIBS potentialities for particle detection [11]. LIBS has already been utilized for on-line monitoring of metal laden aerosols in industrial production, combustion processes [12,13,14], environmental purposes [15,16] and promising results were obtained. More recently, it has been applied to on-line and real time process monitoring during nanoparticle production [17]. LIBS was consequently selected for monitoring purposes near the production processes.

We report in this paper the first results we obtained when applying the LIBS and SMPS systems to nanoparticle detection using two different nanoparticle generators. The goal of the experiments presented in this work was to assess the LIBS potentialities applied to nanoparticle detection in the vicinity of the production processes. The experiments were not only confined to analyzing nanoparticles, namely only those whose sizes are inferior to $100 \mathrm{~nm}$. Particle size range was extended up to $1 \mu \mathrm{m}$-sized particles to better comprehend the mechanisms involved in particle vaporization with the aim in view to eventually gain a better control over the analytical results. In order to make the most of the LIBS experiments, preliminary plasma analysis was done. Time-resolved spectral imagery recordings were performed. The objective was twofold. First, a time resolved 2D mapping allows visualization of the spatio-temporal evolution of the vaporized matter, that is to say, of the exploitable analytical signal for LIBS analyses. Plasma shape and stability were assessed and images were scrutinized to determine whether a preferred spatial location was to be favored when observing the plasma with an optical fiber. Imagery facilitated the selection of the best settings for LIBS signal enhancement. Second, spectral imaging was intended to give a better insight of the mechanisms involved when measuring nanoparticle chemical characteristics utilizing LIBS. Following imagery experiments and taking into account the observations made, the first LIBS experiments were performed on sodium chloride and metallic particles. Particle size dependency on the LIBS signal was investigated irradiating monodisperse particle flows with sizes ranging from $40 \mathrm{~nm}$ to $1 \mu \mathrm{m}$ so as to detect possible impact on the analytical signal. In the course of these experiments, the first values of repeatability and limits of detection were evaluated. Additional experiments aiming at analyzing the analytical signal dependence on plasma temperature were then performed in addition to those already mentioned in order to clarify the mechanisms responsible for high time delays corresponding to LIBS signal emergence. Eventually, the potentialities of LIBS as a tool for workplace surveillance near nanoparticle production processes are discussed. 


\section{Experimental set-up}

\subsection{Description of particle generators}

The general experimental set-up combining the LIBS technique and the SMPS is depicted on figure 1. Two nanoparticle generators were used during our experiments. An atomizer (Constant Output Atomizer Model 3076, TSI) and a spark generator (Aerosol Generator Palas GFG1000) were utilized for particle generation. With both generators, a flow of clean air was obtained connecting the laboratory compressed air line to a filtered air supply unit (TSI Model 3074B).

Using the atomizer, material intended to be generated in aerosol form was dissolved in water (for instance sodium or potassium chloride) and poured into a 1-liter bottle. Two tubes connect the solution contained within the atomizer to the bottle cap: the first from which the solution is drawn from the bottle and the second evacuating excess liquid. Aerosol flow is yielded subsequently to the interaction between a high-velocity clean air jet colliding perpendicularly with the solution being drawn. The aerosol is then flowed through a dryer before entering the LIBS analysis cell or the DMA. Such system produces particles with sizes ranging from a few nanometers to $1 \mu \mathrm{m}$ with a possibility to adjust granulometry by varying the solution concentration.

The spark generator consists of two electrodes whose compositions are that of the material intended to be generated in particulate form. These electrodes are enclosed within a plastic chamber and connected to a capacitance bench fed with a high voltage generator. When operating, the two electrode ends eroded by the successive discharges taking place between them produce particles. These are then evacuated with the clean air flow passing through the electrode interspace. Particle sizes produced with such spark generator vary from a few nanometers to a few hundreds of nanometers.

\subsection{Particle characteristics measurements}

Particle characteristics such as sizes, number and concentration were determined using a Differential Mobility Analyzer (DMA) combined with a particle counter (CPC). The DMA (TSI Series 3080) was employed for two different purposes. First, it was used as a size selector to obtain monodisperse particle flows. Such device permits particle size selection ranging from $10 \mathrm{~nm}$ to $1 \mu \mathrm{m}$ with a resolution of $\Delta d_{p} / d_{p}=0.1\left(d_{p}\right.$ being the measured size). Second, the DMA connected with a particle counter (TSI, CPC Model 3010) allowed to characterize both generators in terms of granulometry and total mass concentration. When determining particle number involving polydisperse flows, another particle counter (CNC GRIM Model 5400) was operated for it made possible the counting of particles whose sizes were inferior to $10 \mathrm{~nm}$. In addition, the total number of 
particles such device can count is higher than that of the TSI CPC described above that is to say $10^{7}$. During particle analysis, the particle flow to be analyzed was divided into two streams the first being directed toward the LIBS analysis cell and the second toward the particle counter using two tubes of equal length. Our study required the analysis of both monodisperse and poydisperse flows. Accordingly, the tube through which particles were flowed was connected either to the outlet of the DMA or to that of the particle generator for monodisperse or polydisperse flow analysis respectively.

\subsection{LIBS and imagery set-up description}

The particles, either metallic or sodium chloride-constituted flowed through the flexible tube connected to the inlet end of the cell. The particle stream was pushed through the tube with a clean air flow rate of 0.7 liters per minute. Under our experimental conditions (inlet and outlet flowrates), the particle distribution was assumed to be quasi homogeneous within the cell where the LIBS analysis was carried out. The outlet end was linked to an extraction pump to evacuate the particles and assure a constant flow rate through the cell. A laser pulse of 5 ns duration and $120 \mathrm{~mJ}$ energy originating from a Q-switched Nd-Yag laser $(\lambda=1064 \mathrm{~nm})$ operated at $20 \mathrm{~Hz}$ was focused inside the cell using a fused silica plano-convex lens of $35 \mathrm{~mm}$ focal length the cell was fitted with. The resulting beam waist value was about $100 \mu \mathrm{m}$ leading to an estimated fluence value of $1500 \mathrm{~J} \mathrm{~cm}^{-2}$ at the focal point. The fraction of the laser beam not absorbed by the plasma was directed toward a beam stop outside the cell through a quartz window being positioned opposite the focusing lens on the other side of the cell. A third quartz window, whose surface is perpendicular to the other two, was used to collect the light emitted by the plasma both for imagery and LIBS purposes in a direction perpendicular to the laser beam. Plasma images were recorded using a fast intensified CCD camera (Andor Istar, model DH734-18F-03) with a Nikkon objective (focal length of $50 \mathrm{~mm}$ ) mounted on three extension ring so as to reach a magnification of $3 / 2$. Sodium chloride spectral imaging recordings were made possible by placing a $10 \mathrm{~nm}$ width interference filter centered on sodium strongest line wavelength $(588.99 \mathrm{~nm})$ in front of the objective. Sets of different optical densities were utilized when necessary and more specifically for plasma image recordings to avoid saturating the ICCD detector. The LIBS signal was collected through the same quartz window with a $50 \mathrm{~mm}$ focal length lens. The plasma emission was imaged onto the entrance of a $50 \mu \mathrm{m}$ diameter core optical fiber with a magnification of $1 / 3$. The fiber was linked to an Echelle spectrometer (Mechelle 5000, resolution of $0.1 \mathrm{~nm}$ at $500 \mathrm{~nm}$ ) equipped with ICCD camera presented above. 


\section{Results and discussion}

\subsection{Imagery}

\subsubsection{Plasma imagery}

Time-resolved plasma imagery experiments on laser-induced sparks generated in air subsequent to single shots were recorded to determine temporal evolution of plasma characteristics such as size, shape and lifetime. Some studies were already made on laser-induced spark breakdown in air [18,19,20,21]. Accordingly, the plasma expansion and its characteristics will be briefly described. Plasma images produced during these experiments when focusing a laser pulse in the air are presented on figure 2 as a function of time delay. Recording parameters such as gate widths and sets of neutral density filters used are indicated on the images. Shortly after the end of the laser pulse, the cone-shaped plasma expands rapidly longitudinally and radially between 0 and $20 \mathrm{~ns}$ with estimated velocities of 80 and $20 \mathrm{~km} \mathrm{~s}^{-1}$ for longitudinal and radial expansion respectively, these values falling to $600 \mathrm{~m} \mathrm{~s}^{-1}$ and $250 \mathrm{~m} \mathrm{~s}^{-1}$ when calculated in the 900 to $1500 \mathrm{~ns}$ temporal interval. From 1500 ns to 4500 ns time delay, the plasma expansion slows down, stabilizes in shape and plasma dimensions are approximately $4.5 \mathrm{~mm}$ long and $2.5 \mathrm{~mm}$ width. Time delay of around ten $\mu$ s marks the beginning of the plasma decay. Its shape modifies with a dramatic decrease in luminosity. Based on these recordings, the plasma lifetime value under our experimental conditions was estimated to be more than $60 \mu \mathrm{s}$.

\subsubsection{Spectral Imaging}

With the aim to understand how the vaporized matter is distributed within the laser-induced plasma plume for analytical measurement purposes, time-resolved spectral imaging experiments were performed while irradiating monodisperse particle flows. This approach was supported by two reasons. First, many nanoparticle production processes are likely to produce monodisperse particle flows or at least particles with a narrow size distribution as their size and shape will define their intrinsic properties. Second, streams of narrow particle size distribution were used to provide a first insight into potential LIBS signal dependency on particle size, this point being intended to be more thoroughly examined in the analytical measurement results. Thus, monodisperse particle streams of $40 \mathrm{~nm}$ and $1 \mu \mathrm{m}$-sized sodium chloride particles were introduced into the analysis cell where the plasma was generated in air at atmospheric pressure. Three series of spectral images were then recorded. Integration times of $1 \mu \mathrm{s}$ were chosen for time delays inferior to $20 \mu$ s and this value was increased to $3 \mu$ s for time delays beyond $20 \mu \mathrm{s}$. The first series was obtained while vaporizing $40 \mathrm{~nm}$-sized particles with a number of particles per cubic centimeters of 50000 whose mass concentration amounted to around $3.7 \mu \mathrm{g} / \mathrm{m}^{3}$. The other 
two series were recorded when irradiating $1 \mu \mathrm{m}$-sized particles for two different mass concentrations of 150 and $1500 \mu \mathrm{g} \mathrm{m}^{-3}$ corresponding to 130 and 1300 particles per cubic centimeters respectively.

Spectral image recordings were acquired using the traditional ensemble averaging method [22] that is to say by accumulating over a large number of laser-induced spark events, namely 200 and 100 for $40 \mathrm{~nm}$ and $1 \mu \mathrm{m}$ sized monodisperse particle flows respectively. Two reasons motivated the use of this procedure. First, when only a few number of particles are vaporized per laser shot, the obtaining of a spectral image with a satisfying signal to background ratio implies to resort to accumulations. Second, analytical measurements whose presentation will follow were intended to be acquired in this way (this choice, not always valid, will then be justified) thus motivating the need to observe temporally-resolved vaporized distribution on the corresponding images. As a consequence, these images do not account for the discrete nature of particles being vaporized at random spots within the laser-induced plasma generated by each shot. They only display spatially-averaged vaporized matter evolution. Single shot experiments aiming at assessing vaporized matter distribution within the plasma for a limited number of particles (i.e. one or more) were only performed on micrometric-sized particles, these having sufficient amount of matter to be single-shot detected.

An additional series of images was recorded with identical accumulation numbers but without particles flowing through the cell so as to assess the plasma continuum emission intensity passing through the interference filter. Three last new series of images were created by subtracting the last series from the other three thereby isolating the spectral signal and yielding the spatiotemporal evolution of the vaporized matter. Figure 3 presents spectral images temporal evolution as a function of time delay.

A first brief examination of these images brings a straightforward remark. The series corresponding to $40 \mathrm{~nm}$ and $1 \mu \mathrm{m}$ monodisperse particle flows display great similarities with one another. At first sight, the spatiotemporal evolution of vaporized matter originating from either $40 \mathrm{~nm}$-sized or $1 \mu \mathrm{m}$ sized particles seems identical. A closer look to spectral images temporal evolution reveals that the emergence of the analytical signal occurs at the same time delay for both $40 \mathrm{~nm}$ and $1 \mu \mathrm{m}$ sized particle flows. Indeed, for time delays inferior to 15 $\mu \mathrm{s}$, both $40 \mathrm{~nm}$ and $1 \mu \mathrm{m}$ sized particle spectral images are identical to plasma emission contribution which suggests that emission originating from sodium chloride is not yet visible. For time delays beyond $15 \mu \mathrm{s}$, vaporized matter emission emerges as the plasma continuum emission starts decaying. In both cases, the emergence of the analytical signal coincides with continuum emission decay as shown by images recorded without particles. Based on image observations, the spectral analytical signal lifetime value is estimated to be greater than $60 \mu$ s taking into account the attenuation caused by the interference filter which optical density 
value is around $10^{4}$. Analytical signal temporal evolution emphasizes the long time delays necessary for detection when analyzing low particle concentration. This point will be examined further in the text when discussing the optical emission spectroscopy results. The peculiar shapes and structures of the spectral images raised questions as to the mechanisms involved when the plasma expands following air breakdown. A previous study carried out by Dors et al. [23] and C.G. Parriger [24] present shadowgraph images recorded when inducing a spark in the air with a $1064 \mathrm{~nm}$ wavelength Nd:YAG laser. Spectral images and shadow graph images show similarities both in shape and in the apparent internal structure of the fluid flow pattern.

\subsubsection{Vaporized matter fluid dynamics}

Plasma shape evolution as a function of time delay observed on our images (figures 2 and 3) was investigated and explained by Dors et al. and Parriger [23,24] using shadow graph recordings and computer simulation. Actually, the large amount of energy deposited in the focal volume results in the generation of a spark driven by strong pressure gradients. As a consequence, a blast wave forms (not visible on our images) and propagates at supersonic speed along with rapid plasma expansion. As the blast wave propagates, the temperature and pressure of the inner gas (between the shockwave front and the plasma front) decreases, to the point the pressure gradient between the shock wave edge and the plasma edge is reversed. This eventually results in an inward propagating gas front moving towards the plasma expanding front. The collapse of this front with the asymmetric conic-shaped plasma front provokes a plasma shape modification. The picture corresponding to a time delay of $4500 \mathrm{~ns}$ on figure 2 shows the beginning of plasma shape modification. From this delay on, the plasma shape evolves and is no longer conical. The rear edge of the plasma (i.e. the plasma edge close to the focal region where the plasma was initiated) is seen to become wider than the front edge (i.e. the plasma edge expanding towards the laser beam). The collapse of the two fronts is responsible for the creation of a vortex at the rear edge of the plasma. The vortex is particularly well revealed by spectral images recorded at $25 \mu$ s time delay on figure 3 where it is clearly observed at the rear edge of the plasma. The low-concentrated vaporized matter appears to act as a revelator of the fluid flow pattern inherent to the laser-induced plasma evolution as a function of time delay. Spectral images corresponding to time delays of 15 and $25 \mu$ s suggest that vaporized matter engulf the plasma core with its velocity field directed toward the laser beam which is consistent with plasma expansion direction. Actually, examining the same images, vaporized matter seems to be set in motion in the vicinity of the vortex. In addition, images on figure 3 recorded with $55 \mu$ s time delay also clearly depict a toroidal ring surrounding the optical axis which seems moving along it first toward the laser (in the same 
movement than the aforementioned vaporized matter) and afterwards toward the rear edge of the plasma. This effect was also observed by Beduneau et al. during their investigations while analyzing laser-induced plasma images [21]. Eventually, these results do not allow concluding as to the time delays needed to vaporize $40 \mathrm{~nm}$ and $1 \mu \mathrm{m}$ sized particles. However, they tend to demonstrate, as hinted above, that vaporized matter spatiotemporal evolution does not depend on particle size for sizes ranging from $40 \mathrm{~nm}$ to $1 \mu \mathrm{m}$ in the range of concentrations investigated under our experimental conditions. This similarity suggests that vaporization occurs long before the observation of the spectral signal is made possible. Actually, Hohreiter and Hahn [25] reported a vaporization time of about $15 \mu$ s for a $2 \mu \mathrm{m}$ sized silica particle. Given that the maximum particle size was $1 \mu \mathrm{m}$ under our experimental conditions, vaporization times are very likely to be much lower than $10 \mu$ s. The issue of vaporization time is of great importance when it comes to choose time delay for LIBS signal recording purposes. Time delays inferior or too close to vaporization times inevitably lead to particle size effect when recording the LIBS signal. This point will be further discussed in section 3.2.2 dealing with particle size effects on LIBS signal. Either originating from vaporization of $40 \mathrm{~nm}$ or from $1 \mu \mathrm{m}$-sized particles, vaporized matter spatiotemporal evolution results in a non uniform analytical signal distribution within the plume, the analytical signal being mainly located along the optical axis. Spectral imagery results bring two comments as to the way analytical measurements (i.e. LIBS measurements) should be done using our experimental set-up. First, the analytical signal collection is likely to be optimized pointing the optical fiber towards the optical axis. Second, measurements involving optical fibers whose core diameters are small compared to plasma sizes require to determine which plasma location must be observed to enhance spectroscopic light collection. Given that our experimental configuration falls in this case, spatially-resolved analytical measurements will therefore be performed to determine which location in the plasma, preferentially along the optical axis, should be imaged with the optical fiber to obtain the best signal to background ratio.

\subsubsection{Single shots}

In order to enhance our comprehension of particle vaporization and ensuing diffusion within the plasma plume, experiments involving single particles vaporized using single shots were carried out. Thus, images corresponding to only one shot vaporizing one or more $1 \mu \mathrm{m}$-sized particles were recorded to ascertain, among other things, possible vaporized matter motion around the vortex. These images eliminate the statistical aspect inherent to ensemble averaging and non-spatially averaged images are obtained leading to direct visualization of laser / particle interaction. The average number of particles within the laser-induced plasma may be assessed by 
measuring particle concentration and assuming a plasma sampling volume of a few cubic millimeters or so (based on plasma images recorded at $1 \mu$ s time delay). Thus, a particle density corresponding to only one particle per plasma volume can be reached by lowering the concentration in the atomizer, leading to one particle sampled by the laser-induced plasma volume. One micrometer-sized particles were chosen, their estimated mass of 1,15 pg being far beyond the LIBS detection limit known to be close to $1 \mathrm{fg}$ for sodium chloride at present time, thereby increasing their probability to be single-shot detected with a satisfactory signal to background ratio. Several spectral images of one particle sampled per plasma volume were recorded as a function of time delay using an integration width of $3 \mu \mathrm{s}$. As established before, the signal emerges for a time delay of about $15 \mu \mathrm{s}$. Examining the pictures, vaporized matter emission stemming from the particles is seen to be randomly distributed on the periphery and within the plasma. This result hints that vaporized matter location and expansion in the plasma plume depends on the plasma region where the $1 \mu \mathrm{m}$ sized particles were atomized. Analyzing certain images of figure 4 , it should be noted that it could hardly be told whether there were one or several particles vaporized within the laser-induced spark. Sampling more than one particle is not to be excluded with the settings used for these experiments. These results are consistent with those found out by Lithgow and Buckley [26] when investigating particle location within plasma. However, carrying on the examination, certain pictures exhibit vaporized matter flowing along a fine curved line (figure $4,35 \mu$ s time delay) around the vortex. One possible explanation could be that the vaporized matter follows a streamline wound around the vortex, thereby proving that vaporized matter circulate through the plasma volume. This observation brings two comments. First, this circulation seems to depend on the plasma region where the particles were vaporized. Vaporized matter located in the plasma front edge is less likely to spread through the plasma plume than matter localized in the rear edge where the atomized species are transported following to vortex formation. Second, as matter circulation seems only to occur in the vicinity of the vortex, the likelihood to fill up the plasma with vaporized analytical signal remains dim when analyzing single particles. This implies an adequate choice for the collection system when sampling only one particle per laser shot.

\subsection{Analytical experiments and results.}

\subsubsection{General considerations}

As already reported above, the ensemble averaging method was used for spectra recording. Many LIBS applications require spectra accumulation to yield satisfying results. However, such procedure is applicable provided that it occasion a gain of intensity, not an increase of the background at the expense of the signal. In 
other words, if only too few individual spectra out of the total number of spectra accumulated contain information, another method allowing discarding spectra free of signal should be favored [22]. Our experiments were well suited to the spectra accumulation mode both for nanoparticles $(\mathrm{d}<100 \mathrm{~nm})$ and sub-micrometric particles $(100 \mathrm{~nm}<\mathrm{d}<1 \mu \mathrm{m})$. Assuming, as stated above, a plasma sampling volume of a few cubic millimeters, there was always more than at least one particle sampled by the plasma given the particle concentrations used in this study when analyzing nanoparticles. Indeed, several nanoparticles must be sampled per laser-induced plasma volume so that their chemical composition be potentially detectable, detection efficiency depending on the light collection configuration. Single nanoparticle detection remains a difficult issue at present time and is rarely achieved for most LIBS optical set-up [27]. One nanoparticle mass is falling well below the current LIBS detection limit of $1 \mathrm{fg}$. To give an order of magnitude, one single $100 \mathrm{~nm}$ sized spherical shaped sodium chloride particle has a total mass of approximately $1 \mathrm{fg}$. These results do apply for particle with sizes superior to $100 \mathrm{~nm}$ provided that there be more than one particle per plasma volume. For $1 \mu \mathrm{m}$ sized particles, ensemble averaging efficiency decreases as the number of particles decreases. Low concentrations can lead to less than one particle per plasma volume. However, as already mentioned in the literature [22], if one particle out of ten is detected, ensemble averaging, though less efficient, is still suitable for LIBS analysis purposes.

The analyte, namely the particles in our study, once sampled by the laser-induced plasma must be detected with the appropriate photon collection system in order to yield the final LIBS spectrum. As explained in the experimental set-up, a $50 \mu \mathrm{m}$ core diameter optical fiber offering the best coupling with the Mechelle spectrometer entrance slit was utilized for these first analytical measurements. The collection system allowed the observation of a $150 \mu \mathrm{m}$ diameter spot on the plasma side. Accordingly, the volume of detection that is to say, the plasma volume location imaged onto the optical fiber entrance is much smaller than the plasma sampling volume. This configuration does not allow spatial integration over a large region of the plasma. Spatial integration is said to reduce analytical signal intensity uncertainty one shot after the other, particularly for singleparticle LIBS measurements. However, this has a lesser impact under our experimental conditions. Ensembleaveraged spectral images have demonstrated that vaporized matter is mainly located along the optical axis, leaving no plasma region without signal in the vicinity of the axis as time elapses with respect to the laser pulse. Furthermore, images corresponding to either single shots or spectra accumulation suggested that part of the vaporized matter would circulate around the vortex fueling the optical axis with signal. Thence, signal collection is likely to be optimized by imaging an observation volume placed on the optical axis, choosing an integration 
width for analytical signal recording of several $\mu$ s and taking into account that a maximum intensity should be reached according to the time delay selected and the plasma region on the axis observed.

Temporally and spatially resolved measurements were therefore carried out along the optical axis to determine which plasma region could yield the best signal-to-background ratio. Temporally-resolved LIBS experiments were carried out for three different observed volumes within the plasma (figure 5). A sub micrometric polydisperse particle flow with a concentration of about $50 \mu \mathrm{g} / \mathrm{m}^{3}$ was introduced into the cell. Recordings started for time delays of $15 \mu \mathrm{s}$ up to $160 \mu \mathrm{s}$ with gate widths of $2 \mu \mathrm{s}$. The spectra were acquired over two hundred laser sparks. Three plasma regions have been observed namely, the front edge of the plasma (i.e. the plasma edge expanding toward the laser beam), the plasma center and finally the rear edge of the plasma (i.e. the plasma edge located beyond the focusing point). The temporal evolution of the LIBS signal intensity as a function of time delay for these three plasma locations is presented on figure 6 . These results indicate that the highest LIBS intensity to background ratio is likely to be obtained when observing the rear edge of the plasma. Large integration width values up to $100 \mu$ s could be selected to record the analytical signal leading to the more intense sodium emission lines. Finally, a time delay of $40 \mu \mathrm{s}$ and an integration width of $100 \mu$ s were chosen for spectrum recordings.

\subsubsection{Particle size dependency on LIBS signal}

Having found the best recording parameters, the next step aimed at clarifying whether potential size effects could occur during LIBS measurements on particles with sizes ranging from $60 \mathrm{~nm}$ to $500 \mathrm{~nm}$. Size effects span incomplete vaporization and possible removal of nanoparticles from the plasma precluding their vaporization. Indeed, Carranza and Hahn demonstrated incomplete vaporization of micrometric-sized particles even for high laser irradiance and concluded the existence of an upper particle size of $2.1 \mu \mathrm{m}$ beyond which vaporization was not completed [28]. They also pointed out that this limit would be strongly dependent on experimental setup and parameters. Moreover, Hohreiter and Hahn raised the hypothesis of possible depletion of nanoparticles from the plasma core during its expansion provoked by high pressure gradients [29]. All these points led us to verify whether the LIBS intensity signal was a linear function of mass, whatever particle sizes may be in the range defined above. Monodisperse streams of particles with sizes ranging from $60 \mathrm{~nm}$ to $250 \mathrm{~nm}$ were successively flowed through the cell. LIBS spectra were recorded using the spatio-temporal parameters determined above. An accumulation number of 10000 was necessary to obtain a spectrum with a good signal to background ratio. In parallel to LIBS analysis, real time monitoring of particle concentration was achieved with 
the aid of the GRIMM particle counter. Following these experiments, the LIBS signal intensity was plotted against the mass concentration for different particle diameters. The mass concentration was calculated as described previously. The resulting LIBS intensity (figure 7) proves to be linear as a function of mass concentration for different particle diameters indicating that no particular particle size effects take place under our experimental conditions. Eventually, this result apparently in contradiction with the two above statements regarding particle size effects is actually quite consistent with them when taking a closer look. First, $500 \mathrm{~nm}$ is the maximum particle size analyzed in this experiment far below the upper size limit referred to above. Though made of sodium chloride instead of silica, this $500 \mathrm{~nm}$ sized particle is very unlikely to be incompletely vaporized given both our linear LIBS response curve and previous results described in the literature [28]. Second, Hohreiter and Hahn [29] reported that size effects over the $30-100 \mathrm{~nm}$ range were likely to take place when analyzing carbonaceous particles at early time delays ( 5 - $10 \mu$ s) closer to vaporization times. In addition, Diwakar et al. [30] evidenced that long delay times (50-60 $\mu$ s) minimized size and mass effects on the LIBS response when analyzing nanometric-sized particles. This again quite fits our results obtained using $40 \mu$ s delay time. Indeed, the latter is much higher than the vaporization times, estimated in our work to be much lower than $10 \mu$ s (section 3.1.3) given particle sizes involved. In addition to these experiments, several measurements were carried out at different times and different days in order to assess repeatability.

\subsubsection{Repeatability}

From all these experiments, the repeatability (RSD) was found to be of around $25 \%$. Variability graph is presented on figure 8. Many studies were devoted to the influence of several ambient parameters on the laserinduced spark at atmospheric pressure [18]. The results demonstrated the lack of sensitivity of the latter on these parameters. In the course of our experiments, the average laser energy was monitored regularly and proved to be stable, though shot-to-shot variations may have occurred. Nevertheless, its possible impact on repeatability was lowered due to the large number of accumulation used to record the spectra. Moreover, imagery showed that single shot laser-induced plasmas were quite stable. Actually, the LIBS intensity depends strongly on the amount of matter vaporized within the laser induced spark. Vaporized matter mass variation is likely to be the major cause of intensity variations for two possible reasons. The first reason is the statistical nature of the plasmaparticle interaction. The number of particle present within the plasma may vary from one shot to another. A second reason for intensity variability, even of greater impact than the latter, is the stability of particle generation. During the LIBS experiments, the particle generation was not always stable. As a consequence, the 
error done when calculating the total number of particle per cubic centimeters to be related to the LIBS intensity is not to be underestimated. Uncertainty when determining concentration is probably the major cause of poor repeatability for many measurements in these experiments.

\subsubsection{Limits of detection}

Limits of detection accessible with the current experimental set-up were explored. Successive sodium chloride monodisperse particle flows were introduced into the analysis cell. The nanoparticle sizes and total mass concentrations were lowered until detection with a large number of accumulations, namely 20000, was no longer possible. Time delay and gate widths of respectively $40 \mu$ s and $100 \mu$ s were chosen. Detection of around 36000 of $40-\mathrm{nm}$ sized particles corresponding to a mass concentration of $2 \mu \mathrm{g} / \mathrm{m}^{3}$ was made possible. The spectrum obtained is presented on figure 9. Following these experiments and using the same methodology, successive polydisperse flows of metallic nanoparticles were also analyzed by LIBS using temporal recording parameters identical to those mentioned above and around 10000 laser shots for ensemble-averaging. Nanoparticle flows $(\mathrm{AlO}, \mathrm{CuO}, \mathrm{TiO})$ generated by the spark generator were on turn introduced into the LIBS analysis cell. The mass concentrations corresponding to the flows of nanoparticles with median sizes inferior to $50 \mathrm{~nm}$ were reduced until their detection was no more achievable. The detection limits obtained are reported in table 1 and compared with current values of occupational exposure limits. Detection limits vary from a few $\mu \mathrm{g}$ per cubic meter for elements such as sodium-chloride to a few hundred of $\mu \mathrm{g}$ per cubic meter for aluminum and titanium. In order to determine whether LIBS could possibly be qualified for workplace surveillance, detection limits of titanium nanoparticle, already largely used and mass produced in the industry, were compared to exposure limits recommended by the HSL (Health and Safety Laboratory, UK) or INRS (Institut National de Recherche et de Sécurité, French Institute for Health and Safety at work, France) [31,32]. So far, concentrations such as 4 milligrams per cubic meter for fine $\mathrm{TiO}_{2}$ corresponding to the respirable fraction for a long term exposure limit (8-hour reference period) are advised for worker protection. The first results obtained are encouraging as detection limits obtained for aluminum; copper and titanium are below the recommended values issued by HSL or INRS. However, detection limits should be improved as exposure limit values will probably be lowered in the near future. 


\subsection{High time delays: role of plasma temperature}

To optimize LIBS results, the dependence of the LIBS signal on background gas, laser wavelength and laser energy was investigated. Polydisperse flows of aluminium particles produced using the spark generator, with sizes inferior to $200 \mathrm{~nm}$, particle median diameter of $30 \mathrm{~nm}$ and a mass concentration of $1.5 \mathrm{mg} / \mathrm{m}^{3}$, were introduced into the LIBS analysis cell. Figure 10a, 10b et 10c show the signal-to-noise ratio temporal evolution (obtained from LIBS lines) recorded for three different background gases, laser wavelengths and laser energies. The gate width was set to $500 \mathrm{~ns}$ and the signal was accumulated over 800 laser shots. The other experimental parameters are indicated on the figures. These curves evidence that time delays corresponding to LIBS signal maximum intensities shift toward longer values when increasing the laser wavelength from $266 \mathrm{~nm}$ to $1064 \mathrm{~nm}$ and the laser energy from 20 to $120 \mathrm{~mJ}$. The same shift was observed when changing from air or nitrogen to argon.

In order to get better understanding of the mechanisms at work, the plasma temperature corresponding to the LIBS signal maximum intensities obtained when irradiating the nanoparticles with successive energy values of 20,50,120 mJ were calculated using argon lines and Boltzmann plots. The list of argon lines used and the resulting plasma temperatures are displayed in table 2 and 3. Plasma temperature values proved to be identical, a result already pointed out by Carranza and Hahn [33]. Injecting more energy results in the increasing of the plasma volume. Initial plasma temperature is not altered but the lower the laser energy, the smaller the plasma volume and therefore the faster the plasma cooling speed. Changing background gas, laser wavelength or laser energy results in the modifying of the plasma temperature evolution and therefore of the kinetics of the LIBS signal. These results suggest that when analyzing nanoparticles vaporized within a laser-induced plasma using optical emission spectroscopy under the conditions presented in this work, namely for particles sizes inferior to $200 \mathrm{~nm}$ and mass concentrations below $1.5 \mathrm{mg} / \mathrm{m}^{3}$, the plasma temperature appears to be a key parameter driving the temporal evolution of the LIBS signal. As a consequence, time delays and gate widths must be chosen accordingly to optimize LIBS recording.

In addition to these experiments, the LIBS signal temporal evolution was studied as a function of the chemical elements the nanoparticles flowing through the LIBS analysis cell were made of. Successive nanoparticle flows of aluminium ( $\mathrm{Al}$ ), barium $(\mathrm{Ba})$, carbon $(\mathrm{C})$, calcium $(\mathrm{Ca})$, chromium $(\mathrm{Cr})$, magnesium $(\mathrm{Mg})$, Sodium $(\mathrm{Na})$, nickel $(\mathrm{Ni})$ and silicon $(\mathrm{Si})$ with particle sizes and mass concentrations below $300 \mathrm{~nm}$ and $1 \mathrm{mg} /$

$\mathrm{m}^{3}$ respectively were introduced in the analysis cell for LIBS signal temporal evolution recording purposes. Flows of particles made of barium, calcium, chromium, magnesium, sodium, nickel and silicon were generated 
using the nebulizer whereas nanoparticles made of aluminum, and carbon were produced using the spark generator. The plasma was induced in the air as a background gas with laser pulses of $120 \mathrm{~mJ}$ at $1064 \mathrm{~nm}$. The gate width of the ICCD was set to $5 \mu \mathrm{s}$. The temporal evolutions of the recorded LIBS signal obtained from raw intensity lines normalized to one another are presented on figure 11a (time delay X-axis). Here again, time delays corresponding to LIBS signal maximum intensities clearly differ from one another, depending on the elements nanoparticles are constituted of. Such observation was already reported by Fisher et al [34] when analyzing metallic particles. Local Thermodynamic Equilibrium (LTE) modeling was done to unravel the main mechanism responsible for such observation. Plasmas induced in the air by a laser at atmospheric pressure are often said to be in LTE state [35]. Theoretical line intensity evolutions as a function of temperature were therefore calculated considering a plasma in LTE state and compared to LIBS experimental graphs, whose temporal axis was converted into a temperature axis using an empirical function as explained below. As relative positions on the temperature axis of temperatures corresponding to line maximum intensities are the only elements of interest, the LIBS intensity equation was reduced to the Boltzmann probability equation writing

$$
P_{i}(T)=f(T) \frac{\exp \left(-E_{i} / k T\right)}{Z(T)}
$$

where $\mathrm{P}$ represents the probability for the energy level $E_{i}$ to be populated, $E_{i}$ the upper energy value corresponding to the considered transition, $k$ the Boltzmann constant, $T$ the plasma temperature, $Z(T)$ is the partition function of the considered element, $f$ represents the neutral atom to ions ratio and writes

$$
f(T)=\frac{N_{I}}{N_{I}+N_{H}}
$$

where $N_{I}$ is the total number of atoms in the neutral state and $N_{I I}$ the total number of firstly ionized ions. This ratio is calculated according to Saha's law

$$
\frac{N_{I I}}{N_{I}}=\frac{2}{N_{e}} \frac{\left(2 \pi m_{e} k T\right)^{3 / 2}}{h^{3}} \frac{Z_{I I}}{Z_{I}} \exp \left(-\frac{E_{\text {ion }}}{k T}\right)
$$

where $N_{e}$ is the electronic density, $m_{e}$ the electron mass, $h$ the Planck constant, $Z_{I I}$ and $Z_{I}$ the partition functions of firstly ionized and neutral species respectively, $E_{i o n}$ being the ionization energy.

Based on previous measurements, the electronic density was assumed to be $10^{17} \mathrm{~cm}^{-3}$. All the $P_{i}(T)$ functions were calculated for all the aforementioned elements and normalized to one another. In order to compare the experimental results to these calculations, that is to say, to compare the temperature values corresponding to the experimental LIBS signal maximum intensities with the temperatures corresponding to the 
line maximum intensities predicted by LTE modeling, an empirical function was utilized to turn the temporal axis of the experimental LIBS recordings into a temperature axis. The empirical function used writes

$$
k T=1.3 t^{-0.2}(\mu s)
$$

The parameters of this function were adjusted so as to verify whether the relative positions on the temperature axis of the temperature values corresponding to maximum line intensities predicted by LTE for each chemical element matched the relative positions on the same axis of temperatures corresponding to experimental LIBS signal maximum intensities. It should be noted that relative positions on the temperature axis of the temperatures corresponding to line maximum intensities are unaltered as this function is monotonously decreasing. In addition, equation 4 leads to temperature values of 1.3 and $0.4 \mathrm{eV}$ for time delays of 1 and $100 \mu \mathrm{s}$ respectively, values in accordance with those found in the literature for temperature temporal evolution of laserinduced plasmas generated in air.

The two series of curves depicting the experimental and LTE-predicted evolutions of ionic and neutral line intensities for different elements are displayed on figure 11a (temperature X-axis) and 11b respectively. Ionic line intensities are found to be strictly decreasing with temperature which seems logical as the ions start recombining into neutrals the moment the laser pulse is turned off. The results obtained for ionic line evolutions either experimental or LTE-predicted are in good agreement in view of the roughness of the theoretical approximations. At first glance, the relative positions on the temperature axis of the temperatures corresponding to line maximum intensities either experimental or LTE-predicted are in the same order. A closer look reveals that the order is respected save for the aluminum line. This highlights the limits of this approach and the fact that such calculations only aim at getting a good insight of the general mechanisms taking place within the plasma and does not allow going into detail. Several possible explanations could account for this odd one out, such as aluminum oxidization or strong self-absorption. However, the above mentioned suggestions could apply to the other elements and therefore do not provide sound arguments. These last experiments emphasize that the LIBS signal maximum intensity appears for a preferential temperature depending on the chemical element nanoparticles are composed of.

Eventually, the experiments carried out in this work demonstrated that the temporal evolution of the LIBS signal varies according to (1) the chemical elements the nanoparticles are made of. Indeed, the line maximum intensity appears at a preferential temperature according to Boltzmann's law. It also varies according to (2) the background gas, the laser wavelength, the laser energy. These parameters influence plasma temperature evolution within which the nanoparticles are vaporized. Actually, the nanoparticles are not 
vaporized by direct laser irradiation but within the laser induced plasma. This can be easily verified by comparing nanoparticle concentration during our experiments with the laser focal volume approximated using the following formula [24]

$$
V=19.2\left(\frac{f}{D}\right)^{4} \lambda^{3}
$$

where $f$ and $D$ are the focal lens length and beam diameter respectively, $\lambda$ the laser wavelength and $V$ the focal volume. The comparison of the approximated focal volume value of $210^{-8} \mathrm{~cm}^{-3}$ with the sodium chloride concentration of 40000 part $/ \mathrm{cm}^{-3}$ measured during one of our experiments (figure 3 ), flow of $40 \mathrm{~nm}$ sized nanoparticles) makes it clear that nanoparticles are mainly vaporized within the laser-induced plasma generated in the background gas. According to the plasma cooling speed, the time necessary for a line of a given element to reach its preferential temperature will impose the time delay for which the LIBS signal is to the maximum. Thus, the slower the plasma cooling speed (for instance by increasing laser energy), the longer the time delays corresponding to LIBS maximum intensities will be. Plasma temperature is therefore one of the key parameter explaining the high time delays observed when analyzing particles with LIBS.

\section{Conclusion}

Experiments aiming at demonstrating the possibility to use LIBS combined with a SMPS as a monitoring tool to detect potential nanoparticle release in the vicinity of the production processes have been carried out. First, spectral imagery experiments were performed to visualize vaporized matter spatio-temporal evolution in order to optimize LIBS measurements. Spectral images have suggested that part of the vaporized matter was set in motion subsequently to vortex formation, the latter physical phenomenon being inherent to laser-induced plasma evolution in air at atmospheric pressure. A great part of the vaporized matter was found to be inhomogeneously localized along the optical axis. Consequently, when imaging a small portion of the plasma onto the optical fiber, a preferential position of the fiber corresponding to the observation of a part of the plasma axis region and maximizing signal collection had to be determined and used for LIBS purposes.

Following imagery experiments, sodium chloride and diverse metallic particles were interrogated by LIBS. The LIBS signal was found independent of particle sizes under our experimental conditions. However, size effects could be noticeable, as indicated by Carranza, Hohreiter and Hahn [28, 29], when vaporizing particles made of elements (for instance carbon) for which LIBS signal maximum intensity is reached for short time delays closer to vaporization times. Recording LIBS signal before vaporization is complete or prior to proper diffusion throughout the plasma volume can lead to non-linear response. The repeatability assessed 
during these measurements was found to be no more than $25 \%$ for the whole campaign of experiments. Sodium chloride nanoparticles of $40 \mathrm{~nm}$ were detected leading to a detection limit corresponding to the estimated mass of this monodisperse particle flow, namely a few micrograms per cubic meters. Detection limits obtained when irradiating polydisperse flow of metallic particles with median sizes below $100 \mathrm{~nm}$ proved to be higher than that of sodium chloride with typical values of a few tens (copper) to a few hundreds (aluminum) of microgram per cubic meters. Though these detection limits are still a bit high, the values found in this study are below the current occupational exposure limits issued by the HSL or INRS, making the LIBS technique a potential candidate for leak detection. However, one could argue that these exposure limits might be revised downwards in the near future as they were not specifically released for nanoparticles. The detection limits found in this study can surely be improved through for instance, an advanced data processing algorithm, an optimized collection system or a more sensitive detection system such as a spectrometer coupled with a photomultiplier, the possibility of using a dedicated detection system centered on a wavelength of interest notwithstanding.

The measurement of nanometric and submicrometric particle characteristics consisting, among other things, to relate the LIBS intensity to mass concentration raises the question of the estimation of the latter. This estimation is usually done assuming spherical-shaped particles and multiplying this spherical volume by the density of the measured element. However, in many cases, morpholgy and density of these particles do not fit theoretical values thus leading to wrong estimation of the mass. These considerations underline the need for a reliable particle generator allowing the generation of precise mass flows intended to be used as calibration standards [36,37]. Generating a nanoparticle mass flow as close as possible to a calibration standards requires a thorough measurement of the mass, the density and the morpholgy of the particles produced which is not so easily achieved. Nevertheless, such experiments are imperative in order to eventually obtain quantitative results from the LIBS experiments.

Spectral imaging or emission spectroscopy analyses presented in this work underlined the high time delays necessary for the analytical signal to emerge when vaporizing particles. To this respect, the role of plasma temperature was investigated. Study of LIBS signal kinetics compared with LTE modeling revealed that LIBS signal maximum intensities are reached for preferential temperatures, these values being different according to the elements the particles are made of. When particles made of a given element are vaporized within the laserinduced plasma, LIBS signal maximum intensity is reached at its preferential temperature corresponding to a given time delay depending on the plasma temperature decreasing speed. The latter proved to be sensitive to changes in background gas, laser wavelength and laser energy. Varying these parameters modifies plasma 
temperature decreasing speed and therefore the optimal time delay corresponding to the LIBS signal maximum intensity recorded when vaporizing particles within a laser-induced plasma.

Eventually, efforts are still to be made to improve detection limits for on-line monitoring, certain elements being more prone to be detected by LIBS than others, depending, among other parameters, on chemical composition. Other LIBS measurements, performed on the production site, consisting of depositing nanoparticles on substrates and interrogating them at regular interval of time, method suitable for chronic risk assessment, have not been carried out yet but could be envisaged in the near future. However, it should be noted that for the time being, individual nanoparticle detection is not feasible with the available technology. Further experiments should confirm that LIBS is probably suitable for in-situ and real-time nanoparticle detection following potential accidental release in the vicinity of nanoparticle production processes.

\section{Acknowledgments}

This work was supported by the Picardie Regional Council (France), the French Ministry of Environment and the FP6-Nanosafe2 European project.

\section{References}

[1] Nanosafe2 newsletter 1 march 2007, www.nanosafe.org

[2] Approaches to safe nanotechnology: an information exchange with NIOSH, version 1.1, July 2006

[3] www.nanosafe.org

[4] A. Franco, S.F. Hansen, S.I. Olsen, L. Butti, Limits and prospects of the "incremental approach" and the European legislation on the management of risks related to nanomaterials, Regulatory Toxicology and Pharmacology 48 (2007) 171-183

[5] P. Fichet, P. Mauchien, C. Moulin, Determination of impurities in uranium and plutonium dioxides by LaserInduced Breakdown Spectroscopy, Appl. Spectrosc. 53 (1999) 1111-1117.

[6] P. Fichet, P. Mauchien, J.F. Wagner, C. Moulin, Quantitative elemental determination in water and oil by laser-induced breakdown spectroscopy, Anal. Chim. Acta 429 (2001) 269-278.

[7] L. Dudragne, Ph. Adam and J. Amouroux, Time-resolved laser-induced breakdown spectroscopy: application for qualitative and quantitative detection of fluorine, chlorine, sulfur and carbon in air, Appl. Spectrosc. 52 (1998) 1321-1327. 
[8] D.A. Cremers and L.J. Radziemski, Detection of chlorine and fluorine in air by laser-induced breakdown spectroscopy, Anal. Chem. 55 (1983) 1252-1256.

[9] D.W. Hahn, M.M. Lunden, Detection and analysis of aerosol particles by laser-induced breakdown spectroscopy, Aerosol Sci. Technol. 33 (2000) 30-48.

[10] L.J. Radziemski, T.R. Loree, D. A. Cremers, N.M. Hoffman, Time-resolved laser-induced breakdown spectrometry of aerosols, Anal. Chem. 55 (1983) 1246-1252.

[11] D.W. Hahn, W.L. Flower, K.R. Hencken, Discrete particle detection and metal emissions monitoring using laser-induced breakdown spectroscopy, Appl. Spectrosc. 51 (1997) 1836-1844.

[12] R.E. Neuhauser, U. Panne, R. Niessner, P. Wilbring, On-line monitoring of chromium aerosols in industrial exhaust streams by laser-induced plasma spectroscopy (LIPS), J. Anal. Chem. 364 (1999) 720-726.

[13] Meng-Dawn Cheng, Field measurement comparison of aerosl metals using aerosol beam focused laserinduced plasma spectrometer and reference methods, Talanta 61 (2003) 127-137.

[14] S.G. Buckley, H.A. Johnsen, K.R. Hencken and D.W. Hahn, Implementation of laser-induced breakdown spectroscopy as a continuous emission monitor for toxic metals, Waste Management 20 (2000) 455-462.

[15] J.E. Carranza, B.T. Fisher, G.D. Yoder, D.W. Hahn, On-line analysis of ambient air aerosols using laserinduced breakdown spectroscopy, Spectrochim. Acta, Part B 56 (2001) 851-864.

[16] G.A. Lithgow, A.L. Robinson, S.G. Buckley, Ambient measurements of metal-containing PM2.5 in an urban environment using laser-induced breakdown spectroscopy, Atmos. Environ. 38 (2004) 3319-3328.

[17] T. Amodeo, C. Dutouquet, F. Tenegal, B. Guizard, H. Maskrot, O. Le Bihan, E. Frejafon, On-line monitoring of composite nanoparticles synthesized in a pre-industrial laser pyrolysis reactor using Laser-Induced Breakdown Spectroscopy, Spectrochim. Acta, Part B 63 (2008) 1183-1190

[18] S. Yalcin, D.R. Crosley, G.P. Smith, G.W. Faris, Influence of ambient conditions on the laser air spark, Appl. Phys. B: Lasers Opt. 68 (1999) 121-130

[19] Y.-L. Chen, J.W.L. Lewis, C. Parriger, Spatial and temporal profiles of pulsed laser-induced air plasma emissions, J. Quant. Spectrosc. Radiat. Transfer 67 (2000) 91-103

[20] N. Glumac, Greg Elliot, The effect of ambient pressure on laser-induced plasmas in air, Optics and Lasers Engineering 45 (2007) 27-35

[21] J.L Beduneau, Y. Ikeda, Spatial characterization of laser-induced sparks in air, J. Quant. Spectrosc. Radiat. Transfer 84 (2004) 123-139 
[22] A. W. Miziolek, V. Palleschi, I. Schechter, Laser-Induced Breakdown Spectroscopy, chapter 5 U. Panne and D. Hahn, Cambridge University Press, 2006.

[23] I.G. Dors and C. Parigger, Computational fluid-dynamic model of laser-induced breakdown in air, Applied Optics, Vol. 42 (30) (2003) 5978-5985

[24] A. W. Miziolek, V. Palleschi, I. Schechter, Laser-Induced Breakdown Spectroscopy, chapter 4 C. Parriger, Cambridge University Press, 2006.

[25] V. Hohreiter and D.W. Hahn, Plasma-Particle Interactions in a Laser-Induced Plasma: Implications for Laser-Induced Breakdown Spectroscopy, Anal. Chem. 78 (2006) 1509-1514

[26] G.A. Lithgow, S.G. Buckley, Influence of particle location within plasma and focal volume on precision of single-particle laser-induced breakdown spectroscopy measurements, Spectrochim. Acta, Part B 60 (2005) 10601069

[27] J.E. Carranza, B.T. Fisher, G.D. Yoder, D.W. Hahn, On-line analysis of ambient air aerosols using laserinduced breakdown spectroscopy, Spectrochim. Acta, Part B 56 (2001) 851-864.

[28] J.E. Carranza and D.W. Hahn, Assessment of the upper particle size limit for quantitative analysis of aerosols using laser-induced breakdown spectroscopy, Anal. Chem. 74 (2002) 5450-5454.

[29] V. Hohreiter and D.W. Hahn, Calibration effects for laser-induced breakdown spectroscopy of gaseous sample streams: analyte response of gas-phase species versus solid-phase species, Anal. Chem. 77 (2005) 11181124

[30] P.K. Diwakar, P.B. Jackson, D.W. Hahn, The effect of multi-component aerosol particles on quantitative laser-induced breakdown spectroscopy: Consideration of localized matrix effects, Spectrochim. Acta, Part B 62 (2007) 1466-1474

[31] List of approved workplace exposure limits (as consolidated with amendments October 2007), EH40/2005 Workplace exposure limits, Health and Safety Laboratory (HSL)

[32] Valeurs limites d'exposition professionnelle aux agents chimiques en France, ED984 june 2008, INRS (Institut National de Recherche et de Sécurité, French Institute for Health and Safety at work, France)

[33] J.E. Carranza and D.W. Hahn, Sampling statistics and considerations for single-shot analysis using laserinduced breakdown spectroscopy, Spectrochim. Acta, Part B 57 (2002) 779-790

[34] B. T. Fisher, H.A. Johnson, S. G. Buckley and D. W. Hahn, Temporal gating for the optimization of laserinduced breakdown spectroscopy detection and analysis of toxic metals, Applied Spectroscopy, 55 (10) (2001) 1312-1319 
[35] D.A. Cremers and L.J. Radziemski, Handbook of laser-induced breakdown spectroscopy, Wiley, 2006.

[36] J.P. Borra, Review Aerosol production and processing by atmospheric Pressure Electrical Discharges: powder production, coatings and filtration, J. Phys. D: Appl. Phys 39 (2006) 19-54

[37] J.P. Borra, A. Goldman, M. Goldman and D. Boulaud, Electrical discharge regimes and aerosol production in point-to-plane DC high-pressure cold plasmas: aerosol production by electrical discharges, J. Aerosol. Sci. 29 (1998) 661-674

\section{Figure captions}

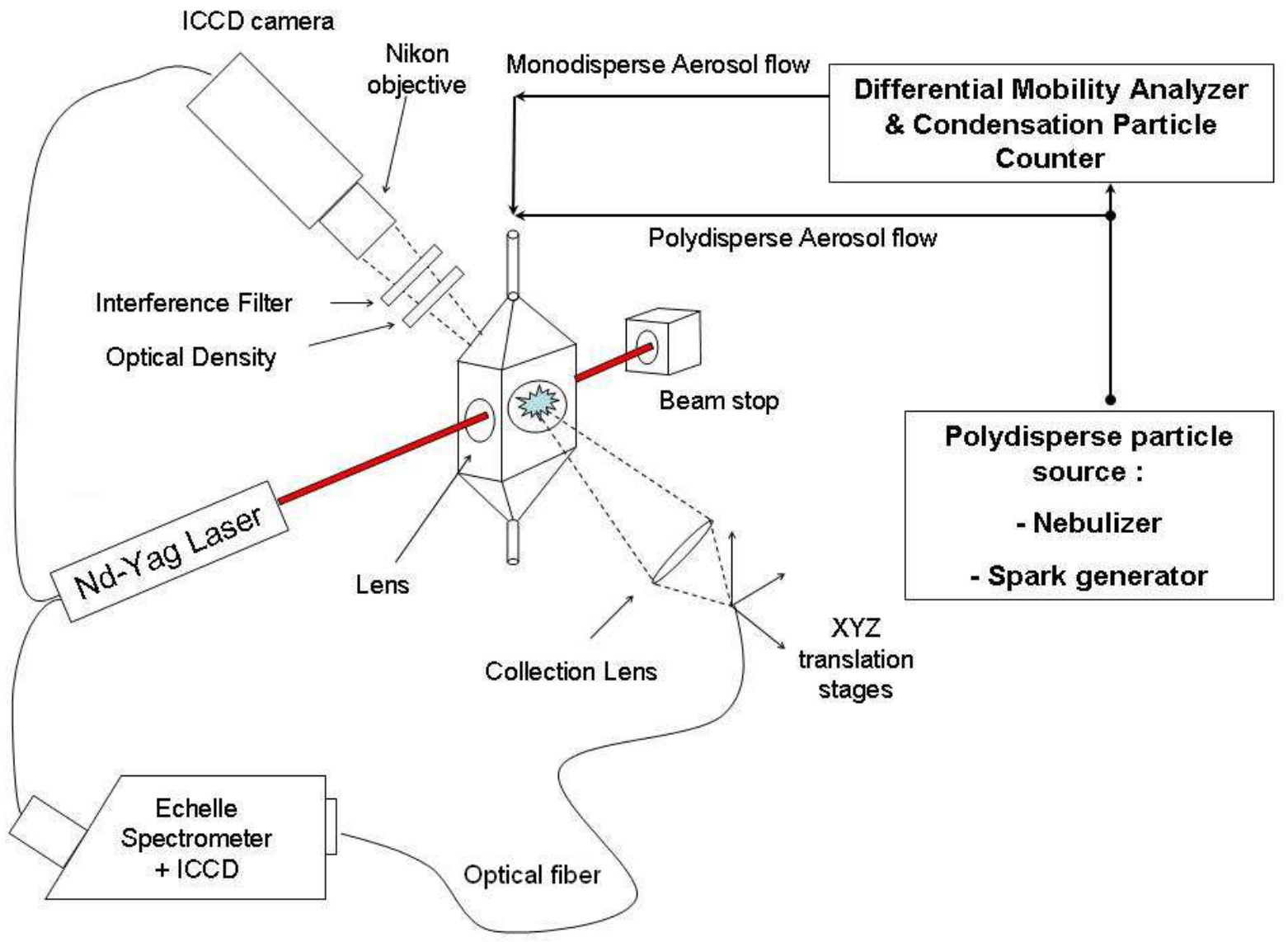

Figure 1: Experimental set-up. 


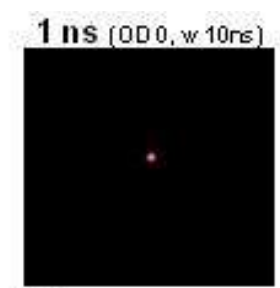

$70 \mathrm{~ns}(0 \mathrm{0} 2.5$, w $10 \mathrm{~ns})$

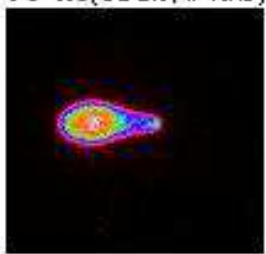

$4500 \mathrm{~ns}(000.5, w 200 \mathrm{~ns})$

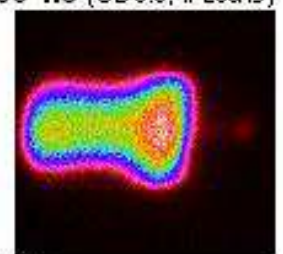

$20000 \mathrm{~ns}(000,100 \mathrm{ac} w 1 \mu \mathrm{s})$

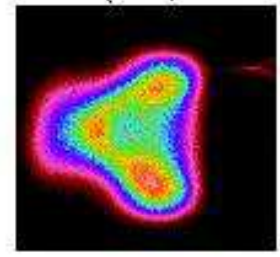

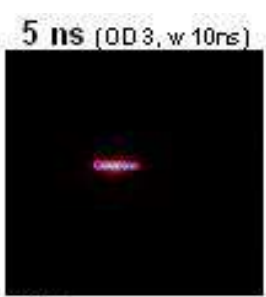

$300 \mathrm{~ns}(001, \mathrm{w} 10 \mathrm{~ns})$

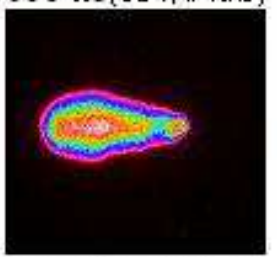

$8000 \mathrm{~ns}(000, w 200 \mathrm{re}]$

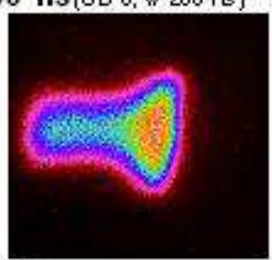

$25000 \mathrm{~ns}(000,100$ ac $w 1 \mu s)$

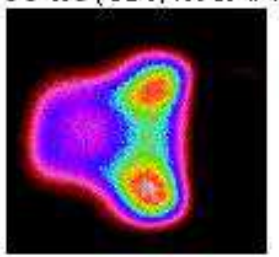

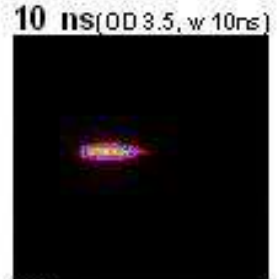

$500 \mathrm{nS}(000.5, w 10 \mathrm{~ns})$

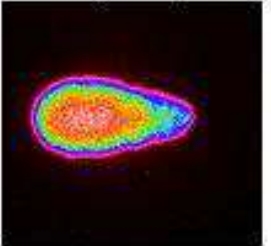

$10000 \mathrm{~ns}[000, w 1 \mu s]$

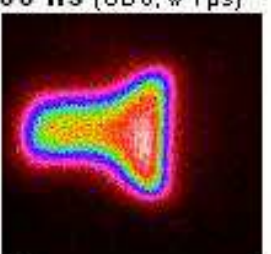

$30000 \mathrm{~ns}(000,100 \approx \times 3 \mu s)$

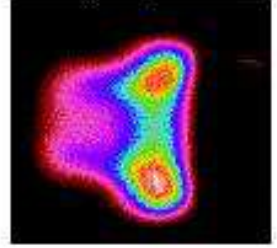

20 ns $\operatorname{con} 3, w 10 n s)$

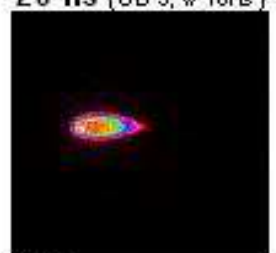

$1500 \mathrm{~ns}(001$, w $200 \mathrm{~ns})$

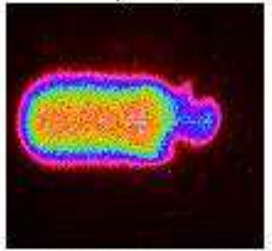

$15000 \mathrm{~ns}[000, w 1 \mu s]$

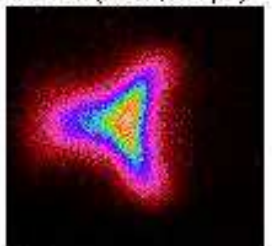

$55000 \mathrm{~ns}(0 \mathrm{D} 0,100 \mathrm{ac} w 3 \mu \mathrm{s})$

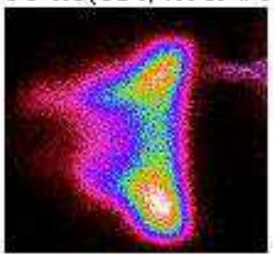

Figure 2. Laser-induced plasma spatio-temporal evolution as a function of time delay (6*6 mm sized). Delays, optical densities (OD) and gate widths are indicated above each image. These images were obtained using single laser shots of $120 \mathrm{~mJ}$ energy igniting plasma sparks in clean air. 


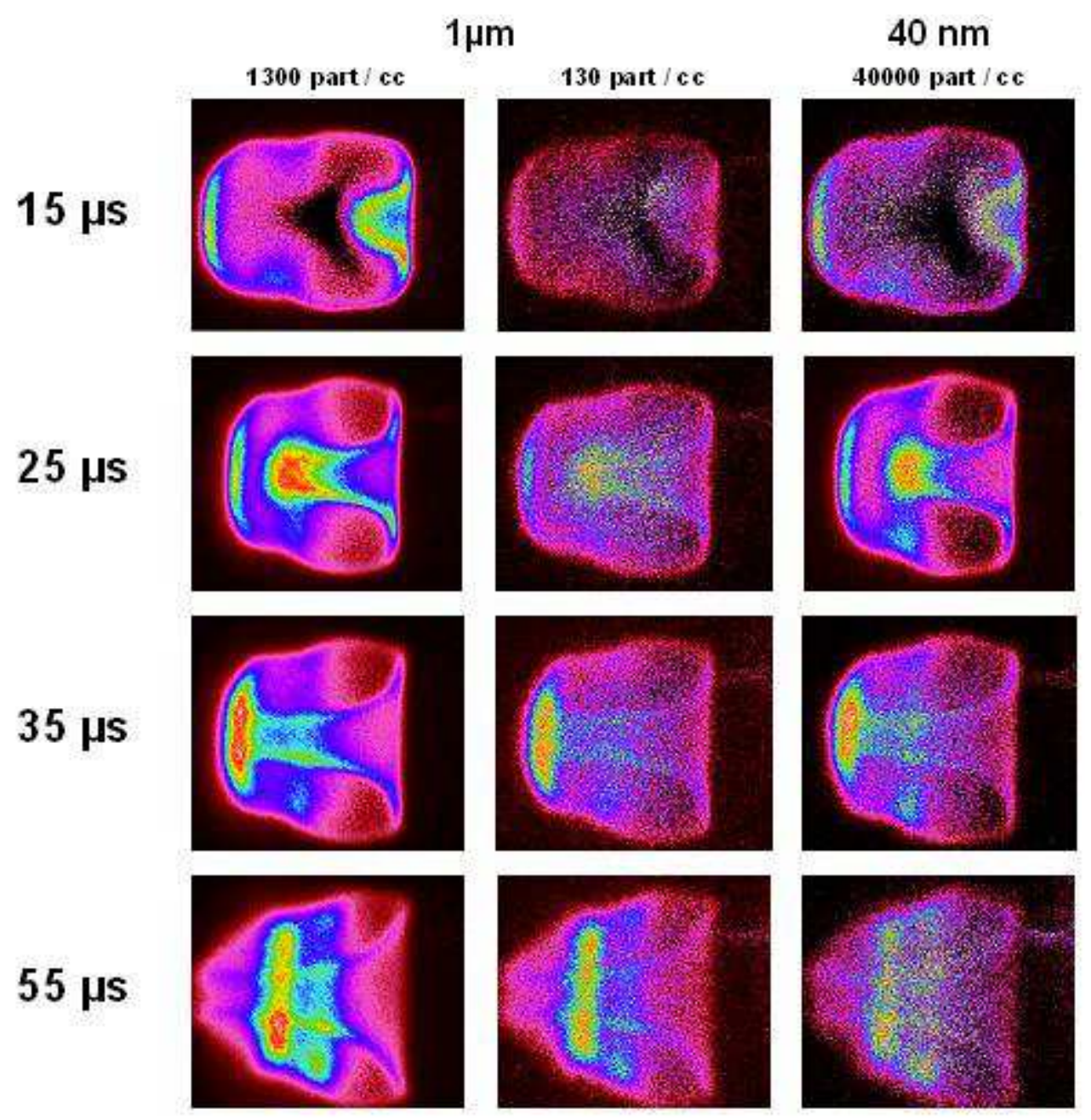

Figure 3. Spatio-temporal evolution of sodium chloride vaporized matter visualized using spectral images acquired over 200 and 100 laser sparks when vaporizing $40 \mathrm{~nm}$ and $1 \mu \mathrm{m}$ sized particles respectively. Time delays and particle numbers are indicated on the figure. Laser energy was set to $120 \mathrm{~mJ}$. 

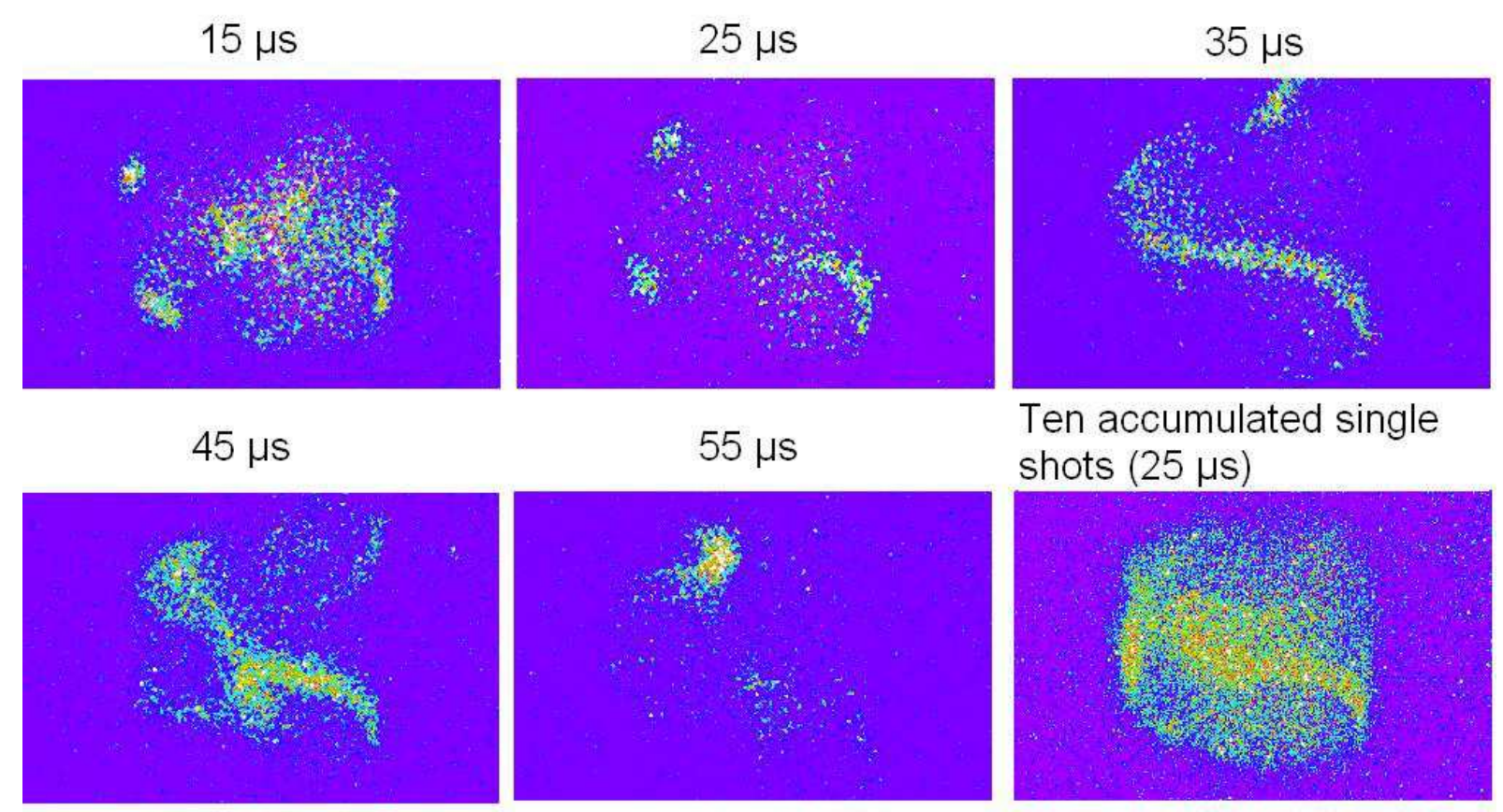

Ten accumulated single shots $(25 \mu s)$

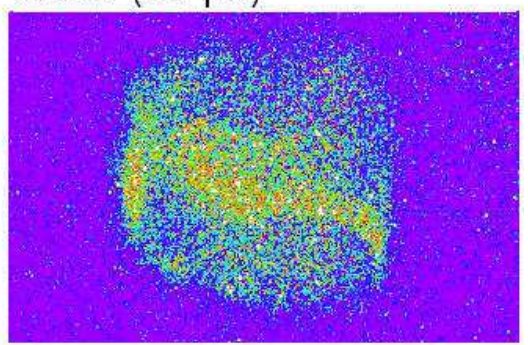

Figure 4. Temporal evolution of analytical signal corresponding to vaporization of at least one $1 \mu \mathrm{m}$-sized sodium chloride particle using single laser shots of $120 \mathrm{~mJ}$ energy. Time delays are indicated above each image and a gate width of $3 \mu$ s was chosen.

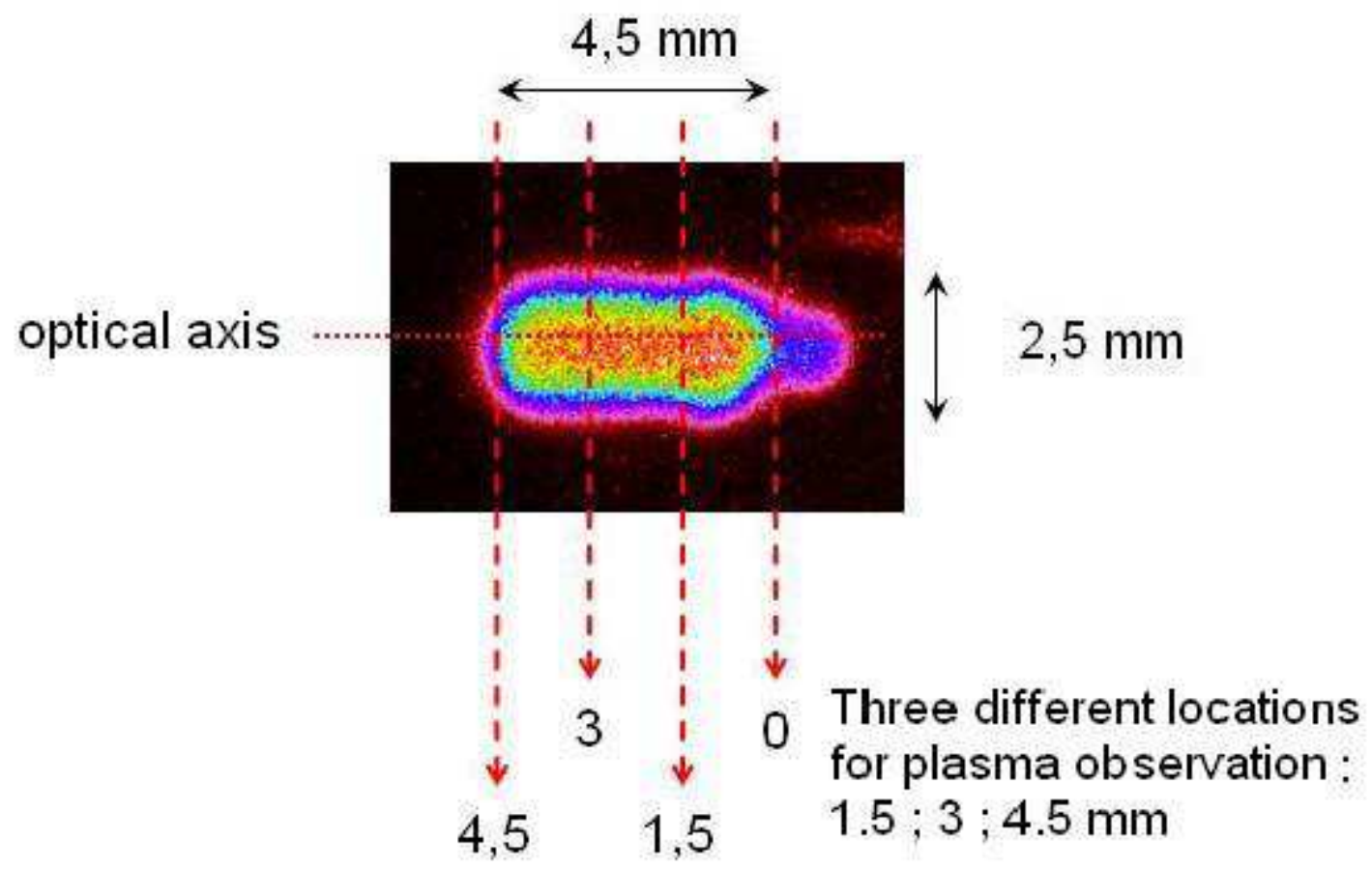

Figure 5. Plasma regions observed with the optical fiber for spatially resolved measurements. 


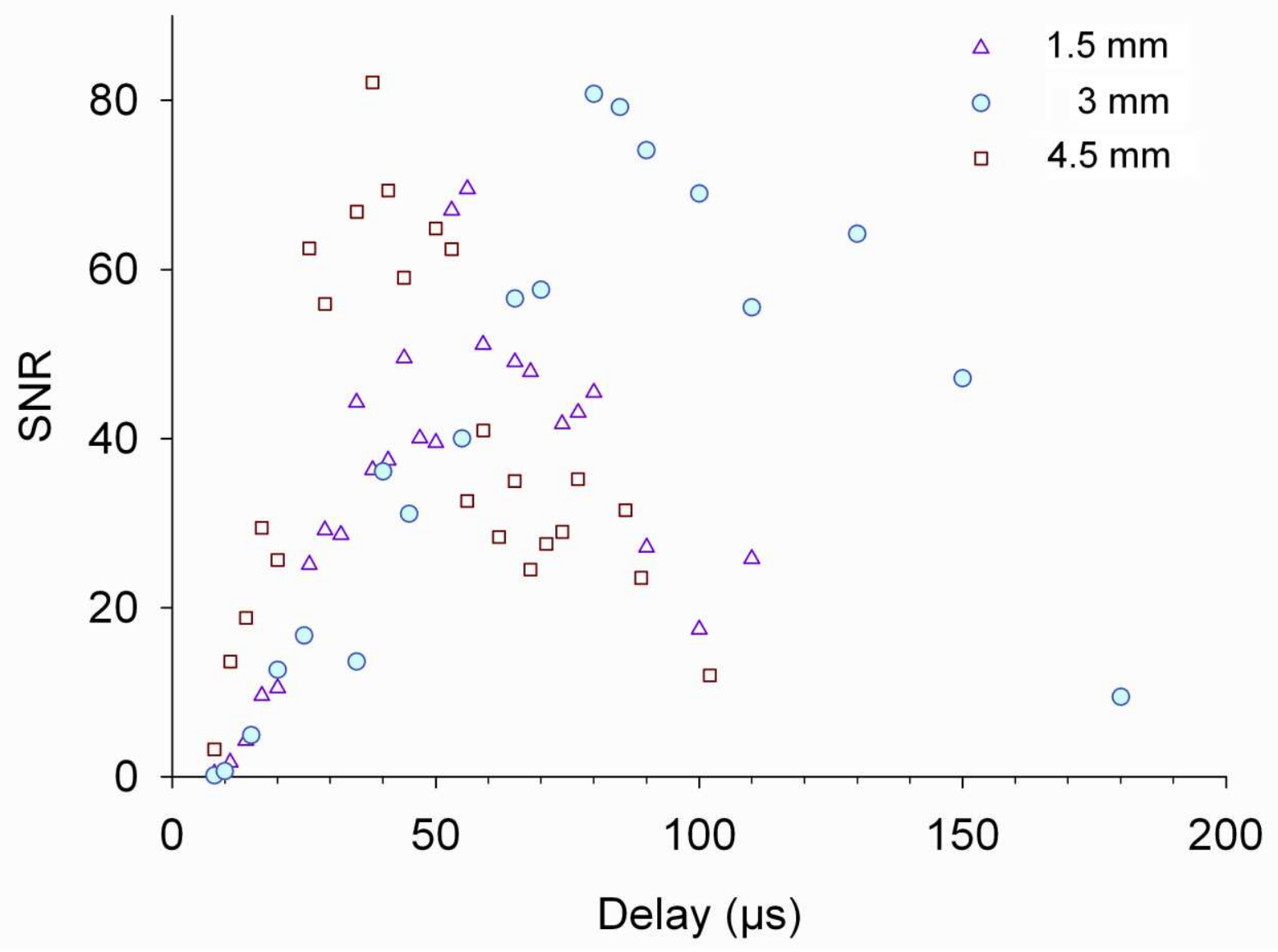

Figure 6. LIBS signal intensity temporal evolution as a function of time delay for three different plasma locations on the optical axis. It was obtained when irradiating a polydisperse flow of sodium chloride particles with a mass concentration of $50 \mu \mathrm{g} / \mathrm{m}^{3}$ using 200 accumulations and a gate width of $2 \mu \mathrm{s}$. The laser energy was set to $120 \mathrm{~mJ}$. 


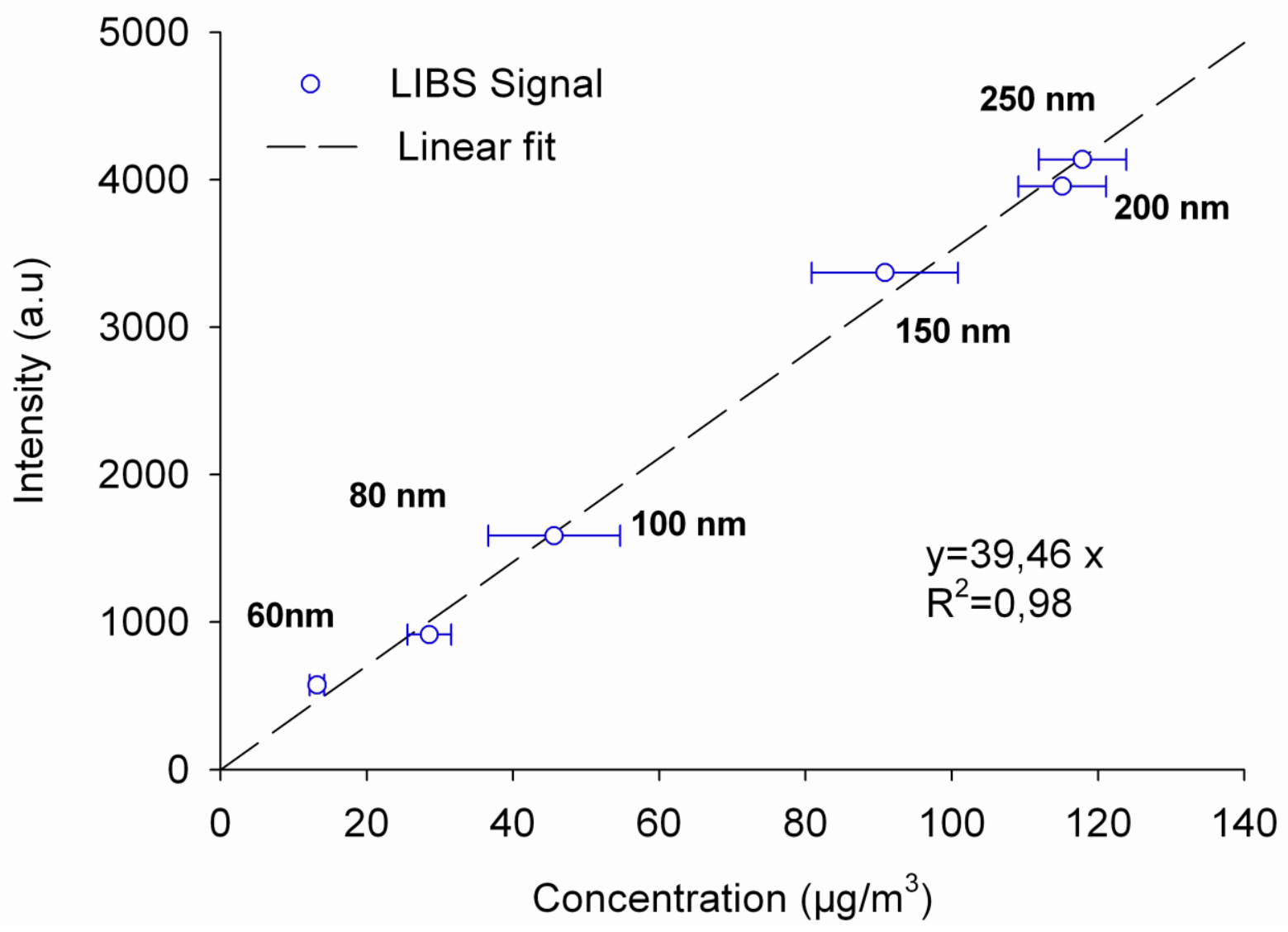

Figure 7. Evolution of LIBS intensity as a function of mass concentration for different particle sizes. It was obtained when irradiating monodisperse flows of sodium chloride particles with sizes ranging from 60 to 250 $\mathrm{nm}$. Time delay of $40 \mu \mathrm{s}$, gate width of $100 \mu \mathrm{s}$ and 10,000 accumulations were used as LIBS experimental parameters. A laser energy of $120 \mathrm{~mJ}$ was utilized. 


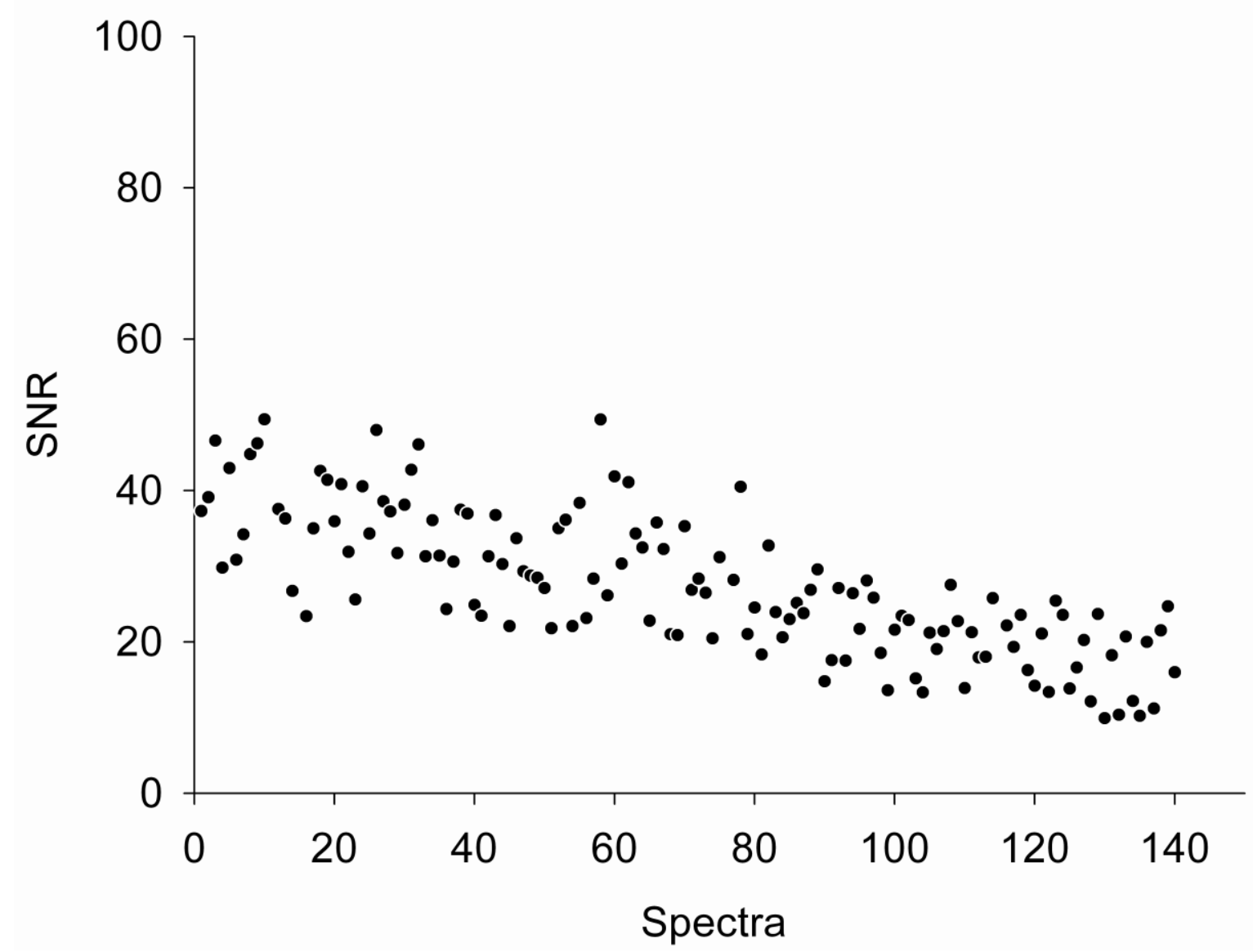

Figure 8 . LIBS signal variability in the course of spectra recording. The drop in particle concentration causes the LIBS intensity to decrease. This plot was obtained when irradiating monodisperse flows of sodium chloride particles with sizes of $400 \mathrm{~nm}$ and particle number varying from 3500 to 1000 particles per cubic centimeters. Time delay of $40 \mu \mathrm{s}$, gate width of $100 \mu$ s and 10,000 accumulations were used as LIBS experimental parameters. The laser energy was set to $120 \mathrm{~mJ}$. 


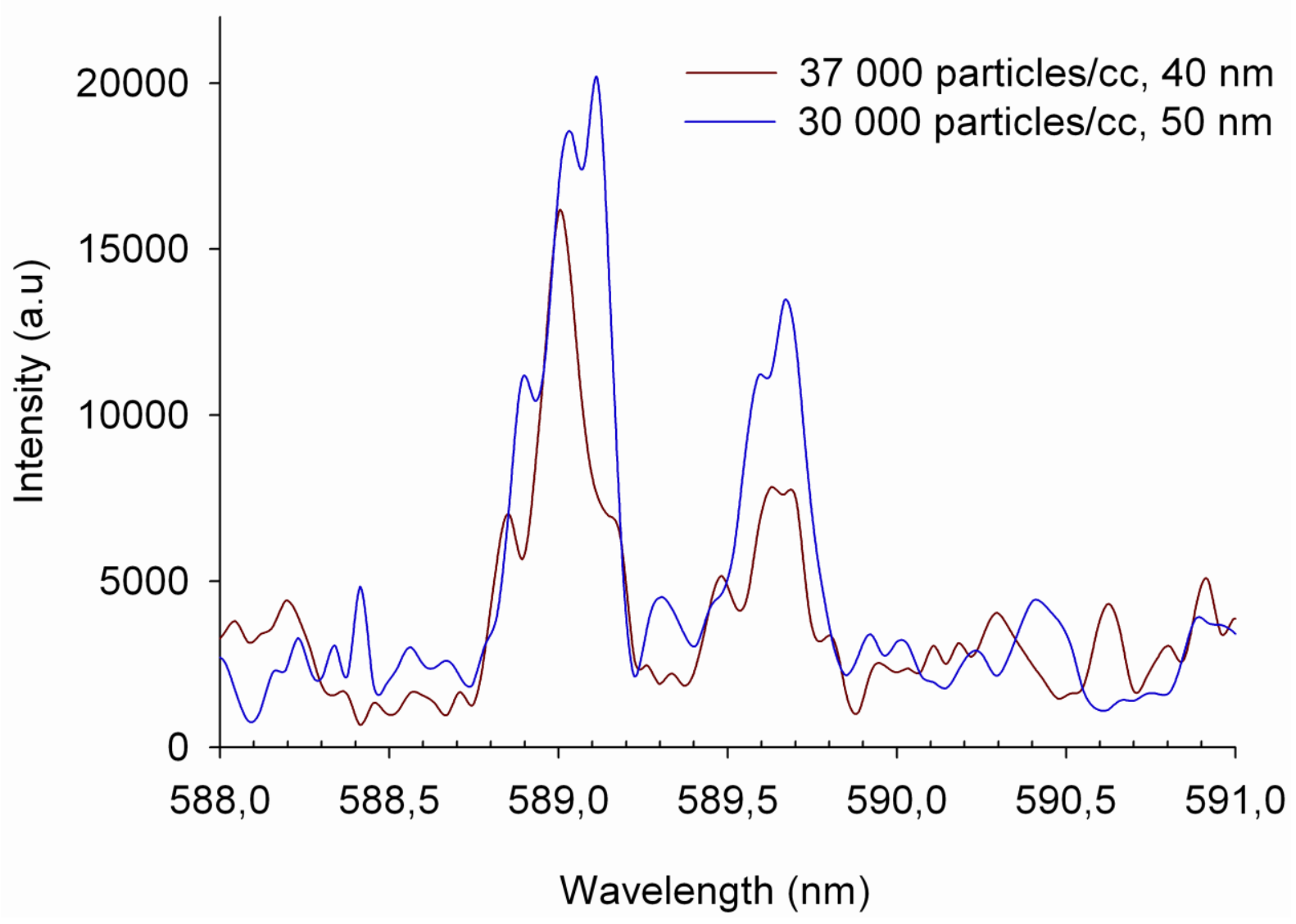

Figure 9. Sodium chloride spectrum corresponding to the best detection limit obtained. The sodium doublet lines were obtained when probing a monodisperse flow of $40 \mathrm{~nm}$ sized particles with number concentration of about 37000 particles per cubic centimeters. Time delay of $40 \mu$ s, gate width of $100 \mu$ s and 20,000 accumulations were used as LIBS experimental parameters. 

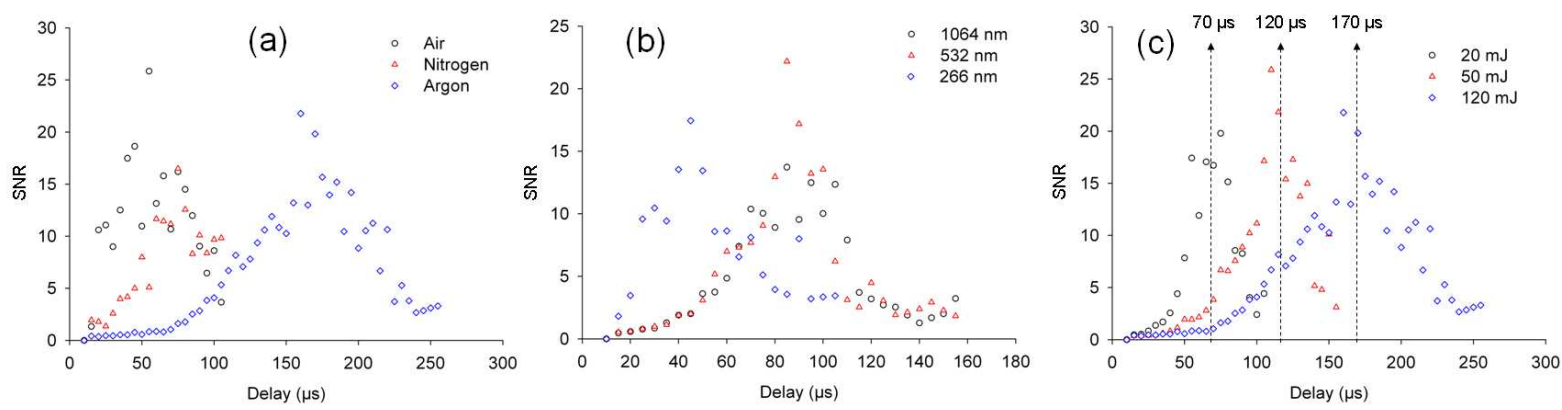

Figure 10. LIBS signal temporal evolution for (a) three different background gas, $\mathrm{E}_{\text {laser }}=120 \mathrm{~mJ}, \lambda=1064 \mathrm{~nm}$ (b) three different laser wavelengths, argon as background gas and $\mathrm{E}_{\text {laser }}=30 \mathrm{~mJ}$ (c) three different laser energies, argon as background gas and $\lambda=1064 \mathrm{~nm}$. These plots were obtained when analyzing polydisperse flows of aluminium particles with sizes ranging from 10 to $200 \mathrm{~nm}$ and a median diameter of $30 \mathrm{~nm}$. Temporal resolution was obtained using $500 \mathrm{~ns}$ gate width and by accumulating spectra over 800 laser shots. 


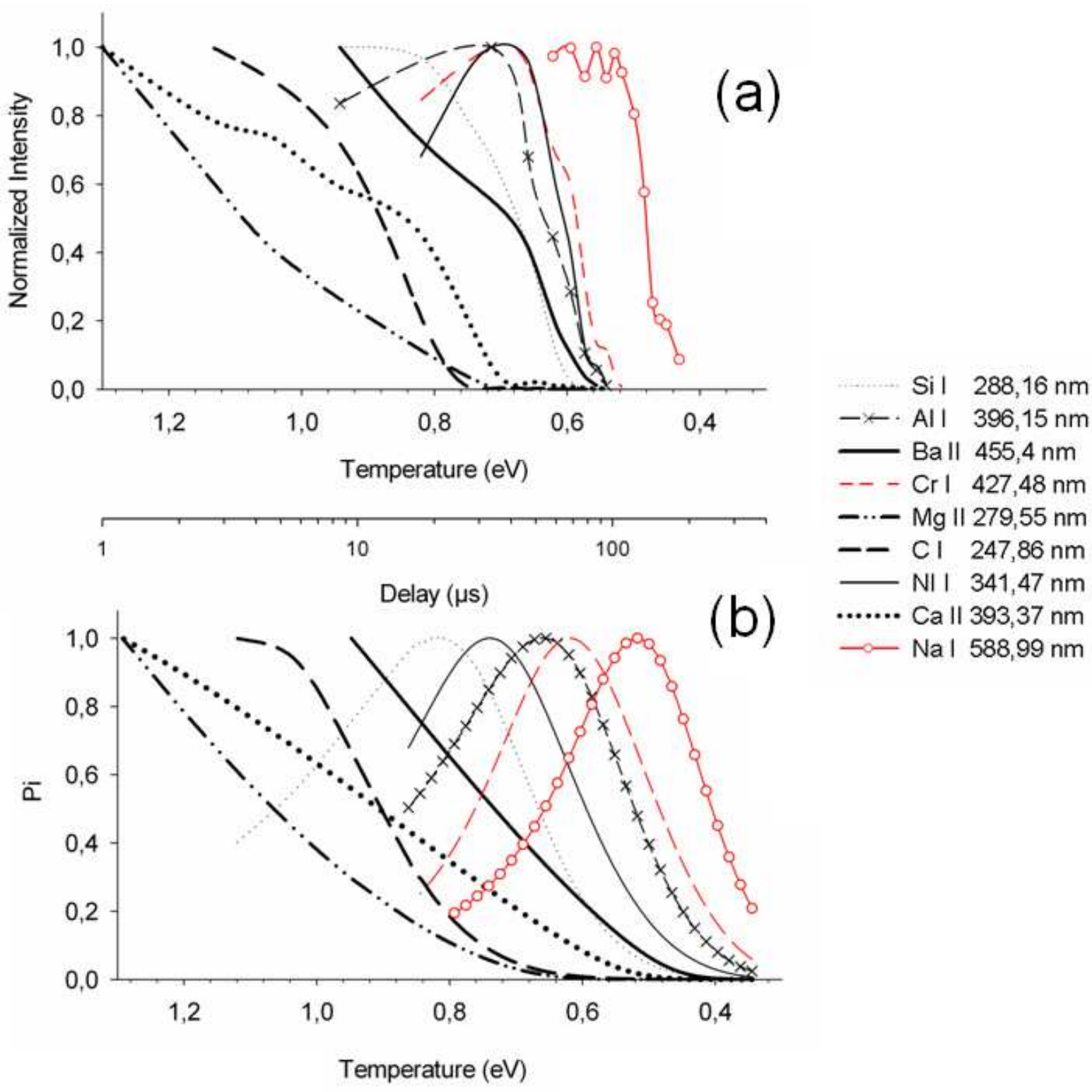

Figure 11. (a) Temporal evolution of normalized LIBS signal (b) Temporal evolution of normalized LTEpredicted intensity line. The experimental LIBS spectra corresponding to the indicated elements were obtained when analyzing polydisperse particle flows with sizes inferior to $300 \mathrm{~nm}$. Laser energy and gate width were set to $120 \mathrm{~mJ}$ and $5 \mu$ s respectively. 


\section{Table captions}

Table 1. Detection limits of a few elements obtained with our LIBS experimental set-up and current occupational exposure limits from HSL [31] and INRS [32]

\begin{tabular}{c|c|c}
\hline Element & Detection limits $\left(\mu \mathrm{g} / \mathrm{m}^{3}\right)$ & Exposure limits $\left(\mu \mathrm{g} / \mathrm{m}^{3}\right)[31,32]$ \\
\hline $\mathrm{Al}$ & 560 & 5000 (fume) \\
$\mathrm{Cu}$ & 80 & 4000 (respirable fraction) \\
& 200 (fume) \\
$\mathrm{Na}$ & 2 & 1000 (respirable fraction) \\
$\mathrm{Ti}$ & 430 & $4000\left(\mathrm{TiO}_{2}\right.$, respirable fraction) \\
\hline
\end{tabular}

Table 2. Wavelengths $\lambda$, transition probabilities $A_{i j} \times 10^{7}$, upper level energies $E_{i}$, and statistical weights of the upper states $\mathrm{g}_{\mathrm{i}}$ of the 28 argon lines selected for the Boltzmann plots.

\begin{tabular}{|c|c|c|c|c|c|c|c|}
\hline$\lambda(\mathrm{nm})$ & $A_{i j}\left(10^{7}\right)$ & $\mathbf{E}_{\mathbf{i}}(\mathbf{e V})$ & $\mathbf{g}_{\mathbf{i}}$ & $\lambda(\mathbf{n m})$ & $A_{i j}\left(10^{7}\right)$ & $\mathbf{E}_{\mathbf{i}}(\mathbf{e V})$ & $\mathbf{g}_{\mathbf{i}}$ \\
\hline 415,86 & 0,14 & 14,53 & 5 & 714,70 & 0,0625 & 13,28 & 3 \\
\hline 418,19 & 0,0561 & 14,69 & 3 & 727,29 & 0,183 & 13,332 & 3 \\
\hline 419,10 & 0,0539 & 14,68 & 3 & 738,40 & 0,847 & 13,30 & 5 \\
\hline 420,08 & 0,0967 & 14,50 & 7 & 750,39 & 4,45 & 13,48 & 1 \\
\hline 425,94 & 0,398 & 14,74 & 1 & 751,46 & 4,02 & 13,27 & 1 \\
\hline 426,63 & 0,0312 & 14,53 & 5 & 763,51 & 2,45 & 13,17 & 5 \\
\hline 427,22 & 0,0797 & 14,52 & 3 & 772,38 & 0,518 & 13,15 & 3 \\
\hline 430,01 & 0,0377 & 14,51 & 5 & 794,82 & 1,86 & 13,28 & 3 \\
\hline 675,28 & 0,193 & 14,74 & 5 & 801,48 & 0,928 & 13,09 & 5 \\
\hline 687,13 & 0,278 & 14,71 & 3 & 810,37 & 2,5 & 13,15 & 3 \\
\hline 693,77 & 0,308 & 14,698 & 1 & 811,53 & 3,31 & 13,08 & 7 \\
\hline 696,54 & 0,639 & 13,33 & 3 & 826,45 & 1,53 & 13,33 & 3 \\
\hline 703,02 & 0,267 & 14,84 & 5 & 840,82 & 2,23 & 13,30 & 5 \\
\hline 706,72 & 0,38 & 13,30 & 5 & 842,46 & 2,15 & 13,09 & 5 \\
\hline
\end{tabular}


Table 3. Plasma temperature calculated for three different delays corresponding to laser energies of 20, 50 and $120 \mathrm{~mJ}$

\begin{tabular}{c|cc}
\hline Laser energy $(\mathbf{m J})$ & Delay $(\boldsymbol{\mu s})$ & Temperature $(\mathbf{K})$ \\
\hline 120 & 105 & 12500 \\
& 170 & 7300 \\
\hline 50 & 75 & 12600 \\
20 & 120 & 7300 \\
\hline & 40 & 12900 \\
& 70 & 7600 \\
\hline
\end{tabular}

